\title{
Multiple-access relaying with network coding: iterative network/channel decoding with imperfect CSI
}

\author{
Xuan-Thang Vu*, Marco Di Renzo and Pierre Duhamel
}

\begin{abstract}
In this paper, we study the performance of the four-node multiple-access relay channel with binary Network Coding (NC) in various Rayleigh fading scenarios. In particular, two relay protocols, decode-and-forward (DF) and demodulate-and-forward (DMF) are considered. In the first case, channel decoding is performed at the relay before $\mathrm{NC}$ and forwarding. In the second case, only demodulation is performed at the relay. The contributions of the paper are as follows: (1) two joint network/channel decoding (JNCD) algorithms, which take into account possible decoding error at the relay, are developed in both DF and DMF relay protocols; (2) both perfect channel state information (CSI) and imperfect CSI at receivers are studied. In addition, we propose a practical method to forward the relays error characterization to the destination (quantization of the BER). This results in a fully practical scheme. (3) We show by simulation that the number of pilot symbols only affects the coding gain but not the diversity order, and that quantization accuracy affects both coding gain and diversity order. Moreover, when compared with the recent results using DMF protocol, our proposed DF protocol algorithm shows an improvement of $4 \mathrm{~dB}$ in fully interleaved Rayleigh fading channels and $0.7 \mathrm{~dB}$ in block Rayleigh fading channels.
\end{abstract}

Keywords: Network coding; Cooperative relaying; Joint network/channel decoding; Imperfect channel state information

\section{Introduction}

In cooperative communications systems, idle nodes have the capability to relay information from other active nodes. Hence multiple copies of the same signal can reach a given destination through independent fading channels, which result in potential spatial diversity gains. However, diversity gains are usually achieved with some loss in system throughput $[1,2]$.

Network Coding (NC) has recently been introduced as a capacity-achieving routing scheme where intermediate network nodes are allowed to combine several input packets into one output packet [3]. Recent results have shown that $\mathrm{NC}$ can also provide improved performance and energy efficiency compared with conventional network routing techniques [4]. However, besides the many

\footnotetext{
*Correspondence: xuanthang.vu@lss.supelec.fr Laboratory of Signals and Systems (LSS), French National Center for Scientific Research (CNRS) - École Supérieure d'Électricité (SUPÉLEC) - University of Paris-Sud 11, 3 rue Joliot-Curie, 91192 Gif-sur-Yvette (Paris), France
}

potential advantages and applications of NC over classical routing, the $\mathrm{NC}$ principle is not without limitations. A fundamental problem that $\mathrm{NC}$ needs to take into account over lossy (e.g., wireless) networks is the so-called error propagation problem: corrupted packets injected by some intermediate nodes may propagate through the network until the destination, and might render impossible to decode the original information $[5,6]$. It is shown in $[5,6]$ that error propagation can dramatically degrade performance and reduce the diversity order of cooperative networks.

Among the solutions that are currently being investigated to counteract the error propagation problem [4], joint network channel decoding (JNCD) is gaining a growing interest [7]. The idea behind JNCD is the exploitation of the inherent redundancy of network and channel codes. In $[7,8]$, it has been shown that, compared to conventional distributed turbo coding and separate network and channel decoding, JNCD can improve the performance of canonical two-way and multiple-access relay channels.

\section{是 Springer}

(c) 2013 Vu et al: licensee Springer. This is an Open Access article distributed under the terms of the Creative Commons

Attribution License (http://creativecommons.org/licenses/by/2.0), which permits unrestricted use, distribution, and reproduction in any medium, provided the original work is properly cited. 
However, these results assume that only correct packets are forwarded from the relay to the destination. Recently, various relaying protocols have addressed the error propagation problem in cooperative communications. In [9-11], the authors propose soft relaying protocols. In softrelaying, the relay does not take any hard decision of the input signals. Instead, the relay computes log-likelihood ratios (LLR) of network-coded bits and re-encodes them using a soft encoder. The relay then forwards encoded soft bits to the receiver. The disadvantage of this method is that it requires higher computational complexity at the relay, as well as larger bandwidth since soft values are transmitted to the destination instead of binary estimates. Another strategy is the so-called threshold-based relaying $[12,13]$ where only decoded bits with reliability above a given threshold are forwarded to the destination. Opportunistic relaying is also useful to combat the error propagation [14]. Opportunistic relaying takes advantage of the many potential relay nodes in the network. The relay with the best end-to-end link is chosen to forward the received data to the destination. It is well-known that error-aware relaying provides better performance than error-unaware relaying protocols. Other solutions foresee that the destination takes care of error propagation. The idea is that, if the destination has access to the channel state information (CSI) of the source-relay links, it can exploit it to counteract the error propagation problem. In [15], the authors show that channel-aware receivers can significantly improve the performance of NC. However, no channel coding is considered in [15]. In [16], a turbolike decoding is proposed. In [17], the authors propose a cooperative communication scheme for multiple-input multiple-output (MIMO) systems. A similar approach is available in [18] without performing channel decoding at the relay.

All these papers assume that CSI and decoding error probability at the relay are available at the destination, which is not always true in practical wireless systems. It is shown in $[19,20]$ that imperfect CSI can significantly degrade the performance of cooperative systems. In this paper, we study the impact of both CSI and decoding error probability at the relay in the multiple access relay channel. It is assumed that CSI at the receivers is acquired via the transmission of pilot symbols. The decoding error probability at the relay is not assumed to be available for free at the destination but we propose a practical way of transmitting a quantized version of it. We study the performance of two notable relaying protocols: Decodeand-Forward (DF) relaying and Demodulate-and-Forward (DMF) relaying. Behind, Compute-and-Forward has been recently introduced as a new relaying protocol which achieves a higher rate than existing relaying techniques and relies on lattice decoding structure [21]. However, this relaying technique is far different from our work in decoding aspects, hence is out of scope of this paper. In DF relaying, channel decoding is performed at the relay before NC and forwarding. On the other hand, in the DMF case, only demodulation is performed at the relay. As such, DMF has less computational complexity than DF but it is more prone to decoding errors at the relay. For each protocol, we develop two new channel-aware JNCD algorithms. To summarize, the contributions of the paper are as follows: we show that JNCD provides better performance than separate network channel decoding only if the destination has enough knowledge of the decoding error probability at the relay; in addition, this gain will be larger as the number of fading blocks per codeword increases. Also, it is shown that the number of pilot symbols mostly affects the coding gain of the system with a negligible impact on the diversity order, at least for the signal-tonoise ratio (SNR) range of interest. Finally, it is shown that CSI quantization errors affect both coding gain and diversity order. Additionally, it is shown that, in general, 3-bit quantization is sufficient for DMF relaying and 6-bit quantization is needed for DF relaying.

The remainder of this paper is organized as follows: section 2 describes system model and notation. Section 3 describes demodulation metrics and channel estimation for imperfect CSI. Section 4 describes the proposed JNCD algorithms. Section 5 describes how to compute the decoding error probability at the relay for various fading situations. Sections 6 and 7 show numerical examples with perfect and imperfect CSIs, respectively. Finally, section 8 concludes this paper.

\section{System model}

The system model under analysis is given by the canonical multiple-access relay channel, where two sources, MS1 and MS2, communicate to a base station (BS) with the help of a relay $R$ [7]. We study the realistic situation where all the channels are subject to Rayleigh fading and additive white Gaussian noise (AWGN). The relay is located between the sources and the base station. We note that in practice, when the relay is very close to the sources, source-relay channels might be subject to different fading models, e.g., rice fading. In this paper, we consider Rayleigh fading assumption for all links for convenience. In order to avoid mutual interference, we consider that transmissions are scheduled in time-orthogonal timeslots [4]. We study both perfect CSI and imperfect CSI at the receiver. Three fading scenarios are investigated: fully interleaved, block fading with $F$ blocks per codeword, and quasi-static fading, i.e., $F=1$.

The source node $\mathrm{MSj}, j \in\{1,2\}$, emits a $K$-length information message $\mathbf{u}_{j}$, where $K$ is the number of information bits in $\mathbf{u}_{j}$. At each source, the information message $\mathbf{u}_{j}$ is processed as follows: (1) first, it is encoded using a recursive convolutional code, which produces a length $N$ 
codeword $\mathbf{c}_{j}$, with $N=K / R$ being the length of the codeword and $R$ being the code rate; (2) then, $\mathbf{c}_{j}$ is interleaved and mapped into a $2^{M}$ constellation point using Gray mapping. This operation provides the modulated signal $\mathbf{x}_{j}$. The modulated signal $\mathbf{x}_{j}$, of length $N / M$ is transmitted to the relay and destination over a Rayleigh fading channel [22] with AWGN. Note that this description involves only the data part. In the imperfect CSI case, we consider that channel estimates are obtained via the use of pilot symbols, and the description will be refined accordingly. These details are provided in the next sections.

We study two relaying protocols: DMF relaying and DF relaying as shown in Figure 1. In DMF relaying, two receivers first demodulate the corresponding signals $\mathbf{y}_{1 r}, \mathbf{y}_{2 r}$ to get the estimated codewords $\mathbf{c}_{1}^{r}, \mathbf{c}_{2}^{r}$. Then the estimated codewords are interleaved before being network-encoded to get $\mathbf{c}_{r}=\pi\left(\mathbf{c}_{1}^{r}\right) \oplus \pi\left(\mathbf{c}_{2}^{r}\right)$, where $\pi$.) denotes interleaving operations and $\oplus$ denotes bitwise XOR operations. In DF relaying, two soft-input hard-output (SIHO) decoders decode $\mathbf{y}_{1 r}, \mathbf{y}_{2 r}$ to get the estimated information messages $\mathbf{u}_{1}^{r}, \mathbf{u}_{2}^{r}$. Note that errors may occur during the decoding process at the relay, i.e., the estimated messages are different from the messages transmitted from the sources. Unlike [12], which only forwards estimated bits with reliability above a certain threshold, we always forward the estimated bits with or without decoding errors (error channel model). However, the receiver will make use of the knowledge of the error probability at the relay. A network encoder encodes the interleaved estimated bits $\pi\left(\mathbf{u}_{1}^{r}\right), \pi\left(\mathbf{u}_{2}^{r}\right)$ to get the network-coded information messages $\mathbf{w}^{r}=\pi\left(\mathbf{u}_{1}^{r}\right) \oplus$ $\pi\left(\mathbf{u}_{2}^{r}\right)$. Then, a channel encoder encodes $\mathbf{w}^{r}$ to get the codeword $\mathbf{c}_{r}$, which is then mapped into the modulated signal $\mathbf{x}_{r}$. The signal received at BS from the sources, at $\mathrm{R}$ from the sources, and at BS from $\mathrm{R}$ are given, respectively, as follows:

$$
\left\{\begin{array}{l}
\mathbf{y}_{\mathrm{jd}}=\sqrt{E s_{\mathrm{jd}}} \mathbf{H}_{\mathrm{jd}} \mathbf{x}_{j}+\mathbf{n}, j=1,2 \\
\mathbf{y}_{\mathrm{jr}}=\sqrt{E s_{\mathrm{jr}}} \mathbf{H}_{\mathrm{jr}} \mathbf{x}_{j}+\mathbf{n}, j=1,2 \\
\mathbf{y}_{\mathrm{rd}}=\sqrt{E s_{r}} \mathbf{H}_{\mathrm{rd}} \mathbf{x}_{r}+\mathbf{n},
\end{array}\right.
$$

where $E s_{j \mathrm{~d}}$ is the energy of the signal received at the destination from $M S j, E s_{j r}$ is the energy of the signal received at the relay from the $\mathrm{MSj}, E s_{r}$ is the energy of the signal received at the destination from the relay. These

a)

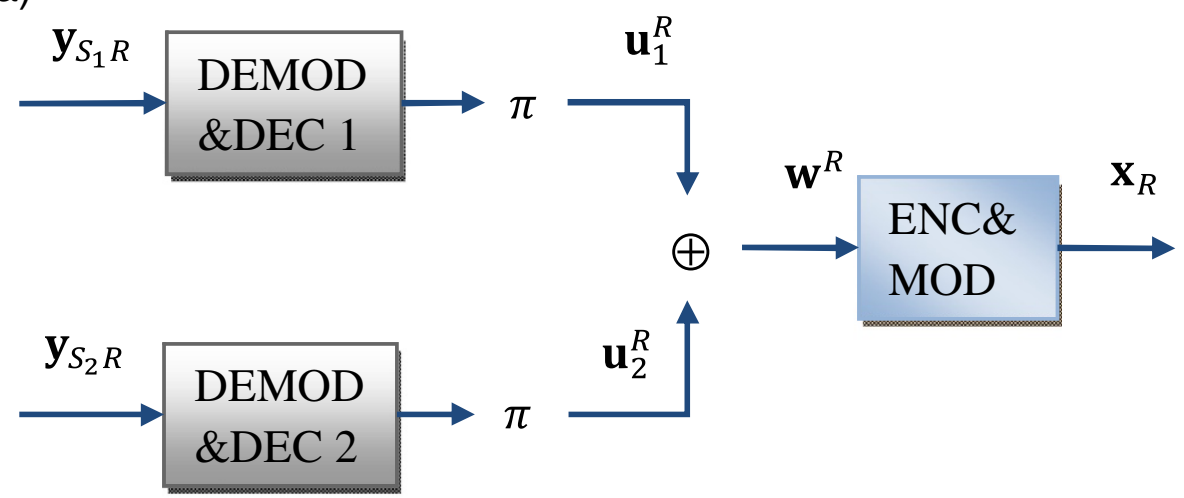

b)
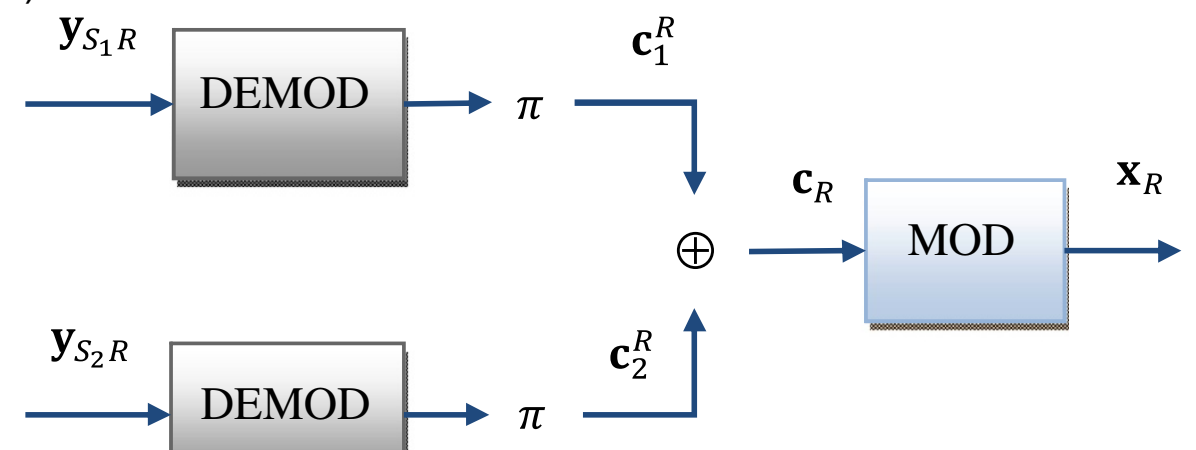

Figure 1 Block diagram of relay and receiver: (a) DF relaying protocol, (b) DMF relaying protocol. 
quantities include the path loss effect; $\mathbf{H}_{\mathrm{jd}}, \mathbf{H}_{\mathrm{jr}}$, and $\mathbf{H}_{\mathrm{rd}}$ are Rayleigh fading coefficient matrices of source-to-relay channels and relay-to-destination channels, respectively, with $E\left[\left\|\mathbf{H}_{(.)}\right\|^{2}\right]=1$. For the sake of simplicity, we use matrix notation $\mathbf{H}_{(.)}$for all fading scenarios considered in this paper. Therefore, the structure of the matrix $\mathbf{H}_{(.)}$ depends on the fading scenario. Three fading channel scenarios are investigated: (1) fully interleaved fading, where $\mathbf{H}_{(.)}=\operatorname{diag}\left(h_{0}, \ldots, h_{N^{\prime}}\right)$, with $N^{\prime}=N / M$, is the length of $\mathbf{x}_{(.)} ;$(2) $F$-block fading, where the number of channel gains in one codeword is equal to $F$, the channel coefficient matrix is of the form $\mathbf{H}_{(.)}=\operatorname{diag}\left(h_{1}, h_{2}, \ldots, h_{F}\right) \otimes \mathrm{I}\left(N^{\prime} / F\right)$, with $\otimes$ denoting the Kronecker product, I $(n)$ being an identity matrix; and (3) quasi-static fading, where we have $\mathbf{H}_{(.)}=h_{0} \times \mathrm{I}\left(N^{\prime}\right)$. Furthermore, $\mathbf{n}$ (index is ignored for simplicity) is the noise vector whose components are circularly symmetric zero-mean complex Gaussian random variables with power spectrum density equal to $\sigma_{n}^{2}, n_{k} \sim$ $\mathrm{CN}\left(0, \sigma_{n}^{2}\right)$.

\section{Channel estimation and modulation metric computation}

This section describes the computation of modulation metrics for both perfect and imperfect CSI as well as how channel estimation for imperfect CSI is performed. These metrics will be used in the next subsections to implement the proposed decoders.

\subsection{Perfect CSI}

For simplicity, we drop the channel indexes in our notation. Let $\mathbf{x}$ and $\mathbf{y}=\mathbf{H x}+\mathbf{n}$ be transmitted and received signals of a generic channel link. The demodulation metric of the $k$ th symbol is computed, given the channel gain $h_{k}$ (corresponding to the $k$ th symbol), as follows:

$$
D_{\mathrm{FCSI}}\left(x_{k}, y_{k} \mid h_{k}\right)=\log \left(\sigma_{n}^{2}\right)+\frac{\left|y_{k}-\sqrt{\operatorname{Es}_{(.)}} h_{k} x_{k}\right|^{2}}{\sigma_{n}^{2}},
$$

where $\mathrm{Es}_{(.)}$is the energy at the destination of the signal received from the sources or from the relay.

Let $C_{k}=\left\{c_{k 1}, c_{k 2}, \ldots, c_{k M}\right\}$ be the $k$ th data symbol, which contains $M$ coded bits, associated to symbol $x_{k}$, belonging to the constellation set $\Theta$. The cardinality of $\Theta$ is equal to $2^{M}$. The a posteriori probability (APP) of the $l$ th bit, $l=1, \ldots, M$ in the $k$ th symbol after demodulating is as follows:

$$
P_{\mathrm{FCSI}}\left(c_{k l}=1\right)=\lambda \sum_{x_{k} \in \Theta, c_{\mathrm{kl}}=1} \exp \left(-D_{\mathrm{FCSI}}\left(x_{k}, y_{k} \mid h_{k}\right)\right),
$$

where $\lambda$ is a normalization factor that satisfies the condition $P_{\mathrm{FCSI}}\left(c_{k l}=1\right)+P_{\mathrm{FCSI}}\left(c_{\mathrm{kl}}=0\right)=1$. Then, the LLR of the coded bit $c_{\mathrm{kl}}, L c_{\mathrm{kl}}$, is:

$$
\begin{aligned}
L c_{\mathrm{kl}} & =\log \frac{P_{\mathrm{FCSI}}\left(c_{\mathrm{kl}}=1\right)}{P_{\mathrm{FCSI}}\left(c_{\mathrm{kl}}=0\right)} \\
& =\log \frac{\sum_{x_{k} \in \Theta, c_{\mathrm{kl}}=1} \exp \left(-D_{\mathrm{FCSI}}\left(x_{k}, y_{k} \mid h_{k}\right)\right)}{\sum_{x_{k} \in \Theta, c_{\mathrm{kl}}=0} \exp \left(-D_{\mathrm{FCSI}}\left(x_{k}, y_{k} \mid h_{k}\right)\right)} .
\end{aligned}
$$

$L c_{\mathrm{kl}}$ is sent to the JNCD decoder and is processed as described in the next sections.

\subsection{Imperfect CSI}

As far as the imperfect CSI case is concerned, we restrict our attention to only block fading channels with $F$ blocks and quasi-static fading with $F=1$. The reason is that channel estimation is assumed to be obtained via a pilotbased approach for [23], which is clearly not compatible with fully interleaved Rayleigh fading. The channel gain is assumed to be constant over one block and is assumed to change independently from block to block. In our setting, a codeword covers $F$ blocks, and the relay estimates the error probability of the whole codeword based on the knowledge of the channel gains of all blocks (see section 5 below). These channel gains are estimated via a pilot message, which is inserted at the beginning of each block, and transmitted via BPSK modulation.

Let $L_{d}$ be the length in bits of the coded data part and $L_{p}$ the length of the overhead. As far as the source-relay links and the source-destination links are concerned, each block consists of $L_{d} / M$ data symbols and $L_{p}$ pilot symbols. As far as the relay-destination link is concerned, the relay also transmits to the destination a quantized version of its decoding error probability which is transmitted in the same way as the pilot bits. In this case, the overhead of length $L_{p}$ consists in the number of symbols $L_{q}$ used for transmitting this error probability (quantization precision), plus the pilot sequence which is thus reduced to $L r_{p}=L_{p}-L_{q}$. In block fading environment with $F>1$, the error probability is concerned with the whole codeword, thus only one block of the R-BS channel is used to transmit this quantized error probability. The packet structure is sketched in Figure 2. Note that pilot and quantization bits are assumed to be binary modulated in order to make the decoding process more robust.

We define a channel rate $R_{c}$ as the ratio of the information bits over the packet length in one channel use (in contrast with standard definition, we include the overhead bits). If $F=1$ and $L_{d}=N$, we have

$$
R_{c}=\frac{R L_{d}}{L_{d}+M L_{p}}=\frac{N R}{N+M L_{p}} .
$$

If $F>1$ and $L_{d}=N / F$, we have

$$
R_{c}=\frac{F R L_{d}}{F L_{d}+M F L_{p}}=\frac{R N}{N+M F L_{p}},
$$

where $R$ is the rate of the channel code and $N$ is the codeword's length. 
a)

$$
\text { S-R, S-D channels }
$$

\begin{tabular}{|c|c|}
\hline$L_{d}$ & $L_{p}$ \\
\hline
\end{tabular}

\section{R-D channel}

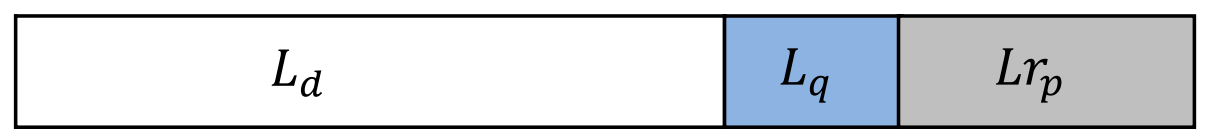

b)

\section{S-R, S-D channels}

\begin{tabular}{|l|l|l|l|l|l|}
\hline$L_{d}$ & $L_{p}$ & $L_{d}$ & $L_{p}$ & $L_{d}$ & $L_{p}$ \\
\hline
\end{tabular}

R-D channel

\begin{tabular}{|l|l|l|l|l|l|l|}
\hline$L_{d}$ & $L_{p}$ & $L_{d}$ & $L_{p}$ & $L_{d}$ & $L_{q}$ & $L r_{p}$ \\
\hline
\end{tabular}

Figure 2 Packet structure for imperfect CSI case: (a) $F=1$ block fading channel, (b) $F=3$ block fading channel.

The difference of the channel rates for $F=1$ and $F>1$ is negligible and can be ignored in practice. For example, for the parameters used in the simulation section, the actual rates for $F=1$ and $F=4$ are respectively 0.476 and 0.417. In this paper, block fading channels with $F>4$ are not considered.

For simplicity, we drop MS and R indexes in our notation. The channel estimation of the generic link works as follows. Each transmission block first consists in the pilot message $\mathbf{x}_{p}$ followed by the data message $\mathbf{x}_{d}$. The power of pilot symbols and data symbols are equal. The corresponding received signals $\mathbf{y}_{p}$ and $\mathbf{y}_{d}$ are of a form as in (1) with only one difference that the channel coefficient $h$ in this case is a scale instead of a vector as in (1).

Note that the use of pilot message and its placement can be optimized via a cross-layer pilot design [24]. In this paper, since we just focus on the impact of imperfect CSI on performance of iterative decoding algorithms, a random sequence is used for pilot message, which obviously is a sub-optimal solution.

A maximum likelihood (ML) estimator is employed. The estimated channel gain is given as [23] $\hat{h}=$ $\mathbf{x}_{p}^{*} \mathbf{y}_{p}\left(\mathbf{x}_{p}^{*} \mathbf{x}_{p}\right)^{-1}$, where (.) $)^{*}$ denotes the transpose conjugate operator, (.) $)^{-1}$, denotes the matrix inverse operator. The channel estimation error is $\tilde{h}=h-\hat{h}$. Employing the mismatch demodulator, the estimated channel coefficient $\hat{h}$ is used as the correct one $h$. The modulation metric is then computed as follows:

$$
D_{\mathrm{PCSI}}\left(x_{k}, y_{k} \mid \hat{h}\right)=\log \left(\sigma_{n}^{2}\right)+\frac{\left|y_{k}-\sqrt{\operatorname{Es}_{(.)}} \hat{h} x_{k}\right|^{2}}{\sigma_{n}^{2}} .
$$

Let $C_{k}=\left\{c_{k 1}, c_{k 2}, \ldots, c_{k M}\right\}$ be the $k$ th data symbol associated to symbol $x_{k}$. The a posteriori probability of the $l$ th bit, $l=1, \ldots, M$ in the $k$ th symbol, $c_{\mathrm{kl}}$, after demodulation can be computed as follows:

$$
P_{\mathrm{PCSI}}\left(c_{\mathrm{kl}}=1\right)=\lambda \sum_{x_{k} \in \Theta, c_{\mathrm{kl}}=1} \exp \left(-D_{\mathrm{PCSI}}\left(x_{k}, y_{k} \mid \hat{h}\right)\right),
$$

where $\lambda$ is a normalization factor such that $P_{\mathrm{PCSI}}\left(c_{\mathrm{kl}}=\right.$ $1)+P_{\mathrm{PCSI}}\left(c_{\mathrm{kl}}=0\right)=1$. The LLR demodulation output of the coded bit $c_{\mathrm{kl}}, L c_{\mathrm{kl}}$ is computed as in (2) with $D_{\mathrm{PCSI}}\left(x_{k}, y_{k} \mid \hat{h}\right)$ is used instead of $D_{\mathrm{FCSI}}\left(x_{k}, y_{k} \mid h\right)$.

These LLR are then used by the JNCD decoder for further processing as described in the next sections.

\section{Proposed joint network/channel decoding}

We propose two JNCD algorithms for the noisy MARC channel. The first algorithm works on possible decoding 
error of the information bits, while the second algorithm works on possible decoding error of coded bits. The error probability of information and coded bits are denoted by $\mathrm{Pe}_{\mathrm{bit}}$ and $\mathrm{Pe}_{\text {code }}$, respectively.

\subsection{Proposed JNCD: Algorithm 1}

The first algorithm is developed based on turbo-like decoding methods. To fully exploit the potential distributed diversity provided by the relay, the destination needs to know the decoding error probability at the relay, which is estimated and transmitted by the relay as described in the previous section. After receiving three channel observations from the two sources and from the relay, along with the decoding error probability at the relay, the destination runs the algorithm as follows:

First, maximum a posteriori probability (MAP) decoding is applied. Let $\hat{\mathbf{c}}_{1}, \hat{\mathbf{c}}_{2}$, and $\hat{\mathbf{c}}_{r}$ be the soft outputs of the demodulators associated to MS1, MS2, and R, respectively. At the destination, the maximum a posteriori probability decision rule is

$$
\widehat{u}_{1 k}, \widehat{u}_{2 k}=\arg \max _{u_{1 k}, u_{2 k}} P\left[u_{1 k}, u_{2 k} \mid \hat{\mathbf{c}}_{1}, \hat{\mathbf{c}}_{2}, \hat{\mathbf{c}}_{r}\right],
$$

where $P[$.$] denotes probability and P[a \mid b]$ denotes probability of $a$ conditioned on $b$.

The probability in (3) is the marginal probability of the whole codeword. With some algebra, (3) can be rewritten as follows:

$$
\begin{aligned}
\widehat{u}_{1 k}, \widehat{u}_{2 k}= & \arg \max _{u_{1 k}, u_{2 k}} \sum_{\substack{\mathbf{u}_{2} \sim\left\{u_{2 k}\right\} \\
\mathbf{u}_{1} \sim\left\{u_{1 k}\right\}}} P\left[\hat{\mathbf{c}}_{1}, \hat{\mathbf{c}}_{2}, \hat{\mathbf{c}}_{r} \mid \mathbf{u}_{1}, \mathbf{u}_{2}\right] \\
= & \arg \max _{u_{1 k}, u_{2 k}} \sum_{\substack{\mathbf{u}_{2} \sim\left\{u_{2 k}\right\} \\
\mathbf{u}_{1} \sim\left\{u_{1 k}\right\}, \mathbf{w}^{r}}} P\left[\hat{\mathbf{c}}_{1}, \hat{\mathbf{c}}_{2}, \hat{\mathbf{c}}_{r} \mid \mathbf{u}_{1}, \mathbf{u}_{2}, \mathbf{w}^{r}\right] \\
& \times P\left[\mathbf{w}^{r} \mid \mathbf{u}_{1}, \mathbf{u}_{2}\right],
\end{aligned}
$$

where $\sum_{\mathbf{u}_{1} \sim\left\{u_{1 k}\right\}, \mathbf{u}_{2} \sim\left\{u_{2 k}\right\}, \mathbf{w}^{r}}$ (.) denotes the sum over all bits of $\mathbf{u}_{1}, \mathbf{u}_{2}, \mathbf{w}^{r}$ except bits $\left\{u_{1 k}, u_{2 k}\right\}$. From (4), we note that, given the information messages, the received signals are independent. Thus, the righthand side of (4) simplifies to:

$$
\sum_{\substack{\mathbf{u}_{2} \sim\left\{u_{2 k}\right\} \\ \mathbf{u}_{1} \sim\left\{u_{2 k}\right\}, \mathbf{w}^{r}}} P\left[\hat{\mathbf{c}}_{1} \mid \mathbf{u}_{1}\right] P\left[\hat{\mathbf{c}}_{2} \mid \mathbf{u}_{2}\right] P\left[\hat{\mathbf{c}}_{r} \mid \mathbf{w}^{r}\right] P\left[\mathbf{w}^{r} \mid \mathbf{u}_{1}, \mathbf{u}_{2}\right] .
$$

The last term in (5) accounts for possible decoding errors at the relay. We note that this decoding error is on the information bits. The related error probability is denoted by $P e_{\text {bit }}$ and it is computed in the next section. We assume, for tractability, that the network-coded information bits are independent (a reasonable assumptions when interleavers at the relay are used). Thus, we have $P\left[\mathbf{w}^{r}\right]=\prod_{k=1}^{K} P\left[w_{k}^{r}\right]$. This assumption leads to a suboptimal JNCD algorithm. In addition, since the transmitted information bits are independent, we have $P\left[\mathbf{w}^{r} \mid \mathbf{u}_{1}, \mathbf{u}_{2}\right]=$
$\prod_{k=1}^{K} P\left[w_{k}^{r} \mid u_{1 k}, u_{2 k}\right]$. Let $w_{k}=u_{1 k} \oplus u_{2 k}$ be the correct network-coded bit. We note that $\mathbf{w}$ is based on the codebook while $\mathbf{w}^{r}$ is based on the actual estimate at the relay. As a result, the decoding error at the relay is determined by $\operatorname{Pr}\left[w_{k}^{r} \mid w_{k}\right]$. The decision rule in (5) becomes

$$
\begin{aligned}
\widehat{u}_{1 k}, \widehat{u}_{2 k}=\arg \max _{u_{1 k}, u_{2 k}} & \left\{\sum_{\mathbf{u}_{1} \sim\left\{u_{1 k}\right\}} P\left[\hat{\mathbf{c}}_{1} \mid \mathbf{u}_{1}\right] \times \sum_{\mathbf{u}_{2} \sim\left\{u_{2 k}\right\}} P\left[\hat{\mathbf{c}}_{2} \mid \mathbf{u}_{2}\right]\right. \\
& \times \sum_{\mathbf{w}^{r}} P\left[\hat{\mathbf{c}}_{r} \mid \mathbf{w}^{r}\right] \times \prod_{l=1}^{K} P\left[w_{l}^{r} \mid w_{l}\right. \\
& \left.\left.=u_{1 l} \oplus u_{2 l}\right]\right\} .
\end{aligned}
$$

The block diagram of the proposed JNCD algorithm is illustrated in Figure 3. The algorithm consists of three SISO decoders for the two sources and the single relay, as well as one network decoder. There is also a decoding check node between the SISO decoder for the relay and the network decoder, which controls the uncertainty of the decoding process at the relay. Let $\mathbf{L} \mathbf{u}_{1}^{\mathrm{Net}}\left(\right.$.), $\mathbf{L} \mathbf{u}_{2}^{\mathrm{Net}}($.), and $\mathbf{L} \mathbf{u}^{\mathrm{Net}}$ (.) be the extrinsic LLRs of the information bits sent by the network decoder to SISO decoder 1, SISO decoder 2, and SISO decoder R, respectively. Also, let $\mathbf{L} \mathbf{u}_{1}^{\text {Dec }}\left(\right.$.), $\mathbf{L} \mathbf{u}_{2}^{\text {Dec }}\left(\right.$.), and $\mathbf{L} \mathbf{u}^{\text {Dec }}($.) be the extrinsic LLRs of the information bits that reach the network decoder from SISO decoder 1, SISO decoder 2, and SISO decoder R, respectively. Furthermore, let $\mathbf{L} \mathbf{u}_{r}^{\operatorname{Dec}}($.$) be the extrinsic$ LLRs of the information bits sent from SISO decoder $\mathrm{R}$ to the decoding check node, and $\mathbf{L u}_{r}^{\mathrm{Net}}$ (.) be the extrinsic LLRs of the information bits that reach the SISO decoder $\mathrm{R}$ from the decoding check node.

The proposed iterative decoding algorithm works by exchanging extrinsic information between the SISO decoders and the network decoder. It consists in the following steps:

Step 0. (Setup) Let $\mathbf{L} \mathbf{c}_{1}, \mathbf{L} \mathbf{c}_{2}$, and $\mathbf{L} \mathbf{c}_{r}$ be the LLRs of codewords $\hat{\mathbf{c}}_{1}, \hat{\mathbf{c}}_{2}$, and $\hat{\mathbf{c}}_{r}$, respectively, which are the outputs of the demodulators described in section 2 . The $k$ th element of $\mathbf{L} \mathbf{c}_{j}, j=1,2, r$ is computed as in section 3 .

Step 1. (Channel decoding) At the $n$th iteration, the SISO decoder $j, j=1,2$, and SISO decoder R run the BCJR algorithm [25], as follows: Input: extrinsic information of coded bits $\mathbf{L} \mathbf{c}_{j}, j=1,2, r$ and a priori information $\mathbf{I A}_{j}^{n}$, Output: extrinsic of information bits $\mathbf{L u}_{j}^{\operatorname{Dec}(n)}\left(u_{j}\right), j=$ 1,2 , and $\mathbf{L} \mathbf{u}_{r}^{\operatorname{Dec}(n)}\left(w^{r}\right)$. The upper index $(n)$ indicates the index iteration. In the first iteration, there is no a priori information for SISO decoders 1,2, and R. 


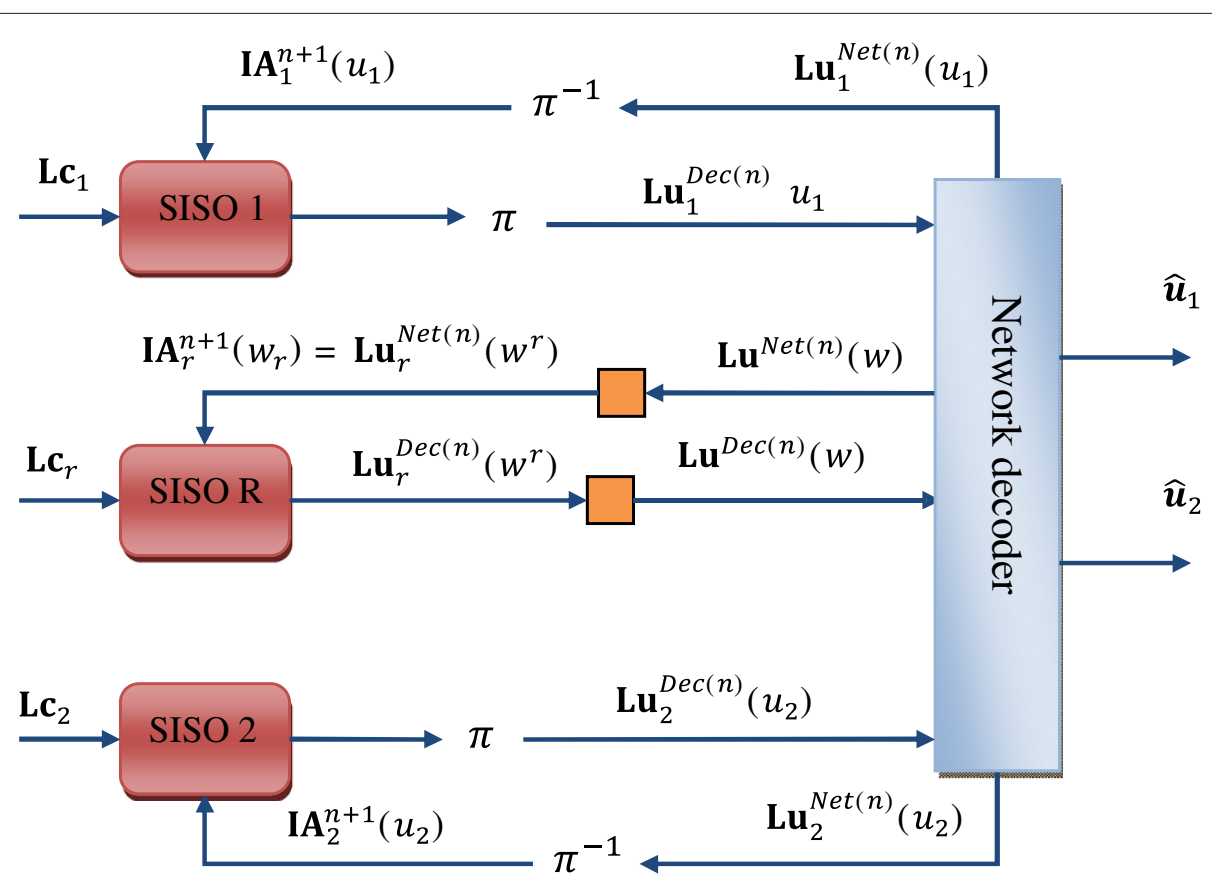

Network check node

Figure 3 Diagram of the proposed JNCD algorithm 1.

Step 2. (Decoding errors are taken into account). The decoding check node updates the extrinsic of the estimated network-coded information bits $\operatorname{Lu} u_{r}^{\operatorname{Dec}(n)}\left(w^{r}\right)$ to get the extrinsic of correct network-coded information bits $\mathbf{L} \mathbf{u}^{\operatorname{Dec}(n)}(w)$ by taking into account the decoding error probability $P e_{\text {bit }}$. Let $\operatorname{Lu}_{r}{ }^{\operatorname{Dec}(\mathrm{n})}\left(w_{k}^{r}\right)$ and $\operatorname{Lu}^{\operatorname{Dec}(n)}\left(w_{k}\right)$ be the $k$ th elements of $\mathbf{L u}_{r}^{\operatorname{Dec}(n)}\left(w^{r}\right)$ and $\mathbf{L u}^{\operatorname{Dec}(n)}(w)$, respectively, then

$$
\operatorname{Lu}^{\operatorname{Dec}(n)}\left(w_{k}\right)=\log \frac{\left(1-P e_{\mathrm{bit}}\right) \exp \left(\operatorname{Lu}_{r}^{\operatorname{Dec}(n)}\left(w_{k}^{r}\right)\right)+\mathrm{Pe}_{\mathrm{bit}}}{\operatorname{Pe}_{\mathrm{bit}} \exp \left(\operatorname{Lu}_{r}^{\operatorname{Dec}(n)}\left(w_{k}^{r}\right)\right)+1-\mathrm{Pe}_{\mathrm{bit}}} .
$$

Step 3. (Network decoding) The extrinsic of information bits $\mathbf{L u}_{1}^{\operatorname{Dec}(n)}\left(u_{1}\right), \mathbf{L u}_{2}^{\operatorname{Dec}(n)}\left(u_{2}\right), \mathbf{L} \mathbf{u}^{\operatorname{Dec}(n)}(w)$ are input to the network decoder to output $\mathbf{L} \mathbf{u}_{1}^{\operatorname{Net}(n)}\left(u_{1}\right), \mathbf{L u}_{2}^{\operatorname{Dec}(n)}\left(u_{2}\right)$, $\operatorname{Lu}^{\operatorname{Net}(n)}(w)$. Let $\operatorname{Lu}_{1}^{\operatorname{Net}(n)}\left(u_{1 k}\right), \operatorname{Lu}_{2}^{\operatorname{Net}(n)}\left(u_{2 k}\right), \operatorname{Lu}^{\operatorname{Net}(n)}\left(w_{k}\right)$ be the $k$ th element of $\operatorname{Lu}_{1}^{\operatorname{Net}(n)}\left(u_{1}\right), \quad \mathbf{L u}_{2}^{\operatorname{Dec}(n)}\left(u_{2}\right)$, $\mathbf{L} \mathbf{u}^{\operatorname{Net}(n)}(w)$, respectively. The outputs of the network decoder are computed as follows:

$\operatorname{Lu}_{1}^{\mathrm{Net}(n)}\left(u_{1 k}\right)=\log \frac{1+\exp \left(\operatorname{Lu}_{2}^{\operatorname{Dec}(n)}\left(u_{2 k}\right)+\operatorname{Lu}^{\operatorname{Dec}(n)}\left(w_{k}\right)\right)}{\exp \left(\operatorname{Lu}_{2}^{\operatorname{Dec}(n)}\left(u_{2 k}\right)\right)+\exp \left(\operatorname{Lu}^{\operatorname{Dec}(n)}\left(w_{k}\right)\right)}$,

$$
\begin{aligned}
& \operatorname{Lu}_{2}^{\operatorname{Net}(n)}\left(u_{2 k}\right)=\log \frac{1+\exp \left(\operatorname{Lu}_{1}^{\operatorname{Dec}(n)}\left(u_{1 k}\right)+\operatorname{Lu}^{\operatorname{Dec}(n)}\left(w_{k}\right)\right)}{\exp \left(\operatorname{Lu}_{1}^{\operatorname{Dec}(n)}\left(u_{1 k}\right)\right)+\exp \left(\operatorname{Lu} \operatorname{Dec}(n)\left(w_{k}\right)\right)}, \\
& \operatorname{Lu}^{\operatorname{Net}(n)}\left(w_{k}\right)=\log \frac{1+\exp \left(\operatorname{Lu}_{1}^{\operatorname{Dec}(n)}\left(u_{1 k}\right)+\operatorname{Lu}_{2}^{\operatorname{Dec}(n)}\left(u_{2 k}\right)\right)}{\exp \left(\operatorname{Lu}_{1}^{\operatorname{Dec}(n)}\left(u_{1 k}\right)\right)+\exp \left(\operatorname{Lu}_{2}^{\operatorname{Dec}(n)}\left(u_{2 k}\right)\right)} .
\end{aligned}
$$

Step 4. (Decoding errors are taken into account) The decoding check node update $\operatorname{Lu}^{\operatorname{Net}(n)}(w)$ to get $\mathbf{L u}_{r}^{\operatorname{Net}(n)}\left(w^{r}\right)$ by taking into account the decoding error probability:

$\operatorname{Lu}_{r}^{\mathrm{Net}(n)}\left(w_{k}^{r}\right)=\log \frac{\left(1-\mathrm{Pe}_{\mathrm{bit}}\right) \exp \left(\mathrm{Lu}^{\mathrm{Net}(n)}\left(w_{k}\right)\right)+\mathrm{Pe}_{\mathrm{bit}}}{\mathrm{Pe}_{\mathrm{bit}} \exp \left(\operatorname{Lu}^{\operatorname{Net}(n)}\left(w_{k}\right)\right)+1-\mathrm{Pe}_{\mathrm{bit}}}$.

Step 5. (Feedback) The extrinsic of information bits $\mathbf{L u}_{1}^{\operatorname{Net}(n)}\left(u_{1}\right), \mathbf{L u}_{2}^{\operatorname{Net}(n)}\left(u_{2}\right)$, and $\mathbf{L} \mathbf{u}^{\operatorname{Net}(n)}(w)$ are feedback to SISO decoders 1,2 , and $\mathrm{R}$ as a priori information for the next iteration, as follows: $\mathbf{I A}_{1}^{n+1}\left(u_{1}\right)=\mathbf{L} \mathbf{u}_{1}^{\mathrm{Net}(n)}\left(u_{1}\right)$; $\mathbf{I} \mathbf{A}_{2}^{n+1}\left(u_{2}\right)=\mathbf{L u}_{2}^{\operatorname{Net}(n)}\left(u_{2}\right) ; \mathbf{I} \mathbf{A}_{r}^{n+1}\left(w^{r}\right)=\mathbf{L u}_{r}^{\operatorname{Net}(n)}\left(w^{r}\right)$.

Step 6. Repeat from Step 1.

\subsection{Proposed JNCD: Algorithm 2}

Algorithm 1 performs channel decoding first and utilizes the decoding error probability of the information bits 
$\mathrm{Pe}_{\text {bit }}$. On the other hand, the second proposed JNCD algorithm exploits the decoding error probability of the coded bits $\mathrm{Pe}_{\text {code }}$ and performs network decoding first. After receiving three channel observations from the two sources and the single relay, along with the decoding error probability $\mathrm{Pe}_{\text {code }}$, the destination applies the MAP decoding rule as follows [26]:

$$
\begin{aligned}
\widehat{\mathbf{u}}_{1}, \widehat{\mathbf{u}}_{2}= & \arg \max _{\mathbf{u}_{1}, \mathbf{u}_{2}} P\left[\mathbf{u}_{1}, \mathbf{u}_{2} \mid \hat{\mathbf{c}}_{1}, \hat{\mathbf{c}}_{2}, \hat{\mathbf{c}}_{r}\right] \\
\sim & \arg \max _{\mathbf{u}_{1}, \mathbf{u}_{2}} \sum_{\mathbf{c}_{1}, \mathbf{c}_{2}} P\left[\mathbf{u}_{1} \mid \mathbf{c}_{1}\right] P\left[\mathbf{u}_{2} \mid \mathbf{c}_{2}\right] \\
& \times P\left[\hat{\mathbf{c}}_{1}, \hat{\mathbf{c}}_{2}, \hat{\mathbf{c}}_{r} \mid \mathbf{c}_{1}, \mathbf{c}_{2}\right] \\
\sim & \arg \max _{\mathbf{u}_{1}, \mathbf{u}_{2}} \sum_{\mathbf{c}_{1}, \mathbf{c}_{2}} P\left[\mathbf{u}_{1} \mid \mathbf{c}_{1}\right] P\left[\mathbf{u}_{2} \mid \mathbf{c}_{2}\right] \\
& \times P\left[\hat{\mathbf{c}}_{1} \mid \mathbf{c}_{1}\right] P\left[\hat{\mathbf{c}}_{2} \mid \mathbf{c}_{2}\right] \times \sum_{\mathbf{c}^{r}} P\left[\hat{\mathbf{c}}_{r} \mid \mathbf{c}^{r}\right] P\left[\mathbf{c}^{r} \mid \mathbf{c}_{r}\right. \\
\triangleq & \left.\mathbf{c}_{1} \oplus \mathbf{c}_{2}\right],
\end{aligned}
$$

where $\mathbf{c}_{j}, j=1,2$ is the codeword generated from the information message $\mathbf{u}_{j} ; \mathbf{c}^{r}$ is the network-coded codeword; $\mathbf{c}_{r} \triangleq \mathbf{c}_{1} \oplus \mathbf{c}_{2}$ is the correct network-coded codeword; $\hat{\mathbf{c}}_{1,2, r}$ is the soft output of the demodulator related to sources 1, 2, and relay R. We note that the correct network-coded codeword $\mathbf{c}_{r}$ is computed from the codebook, while the network-coded codeword $\mathbf{c}^{r}=\pi\left(\mathbf{c}_{1}^{r}\right) \oplus$ $\pi\left(\mathbf{c}_{2}^{r}\right)$ is computed from the estimated codeword $\mathbf{c}_{1}^{r}, \mathbf{c}_{2}^{r}$ at the relay. The two first factors in (10) account for two channel decoders, and the other terms account for the network decoder. The last factor in (10) shows how error decoding on the coded bits at the relay, whose probability is $\mathrm{Pe}_{\text {code }}$, is taken into account by the decoder. The block diagram of this algorithm is sketched in Figure 4. The main difference between algorithm 1 and algorithm
2 is that in the latter case, network decoding is performed first. As a result, one channel decoder can be avoided in algorithm 2, which makes the receiver simpler to implement.

Let $\mathbf{L} \mathbf{c}_{1}, \mathbf{L} \mathbf{c}_{2}$, and $\mathbf{L} \mathbf{c}_{r}$ be LLR inputs for sources 1, 2, and relay $R$, respectively. Let $\mathbf{L} \mathbf{c}_{1}^{\mathrm{Net}}$ and $\mathbf{L} \mathbf{c}_{2}^{\mathrm{Net}}$ be the extrinsic information outputs of the network decoder, and $\mathbf{L} \mathbf{c}_{1}^{\mathrm{Dec}}$ and $\mathbf{L} \mathbf{c}_{2}^{\text {Dec }}$ be extrinsic information outputs of the coded bits of SISO decoder 1 and SISO decoder 2. Finally, let I $\mathbf{A}_{1}$ and $\mathbf{I A}_{2}$ be the a priori information (on coded bits) of the network decoder.

Algorithm 2 consists of following steps:

Step 0. (Setup) The three demodulators process the received signal to output $\mathbf{L} \mathbf{c}_{1}, \mathbf{L} \mathbf{c}_{2}, \mathbf{L} \mathbf{c}_{r}$. The $\mathrm{k} t h$ element of $\mathbf{L} \mathbf{c}_{j}, j=1,2, r$ is computed as in Section 3.

A decoding check node updates $\mathbf{L} \mathbf{c}_{r}$ by taking into account the decoding error probability at the relay, $\mathrm{Pe}_{\text {code }}$, to get $\widetilde{\mathbf{L} \mathbf{c}_{r}}$ :

$$
\tilde{L c}_{r k}=\log \frac{\left(1-\mathrm{Pe}_{\text {code }}\right) \exp \left(L c_{r k}\right)+\mathrm{Pe}_{\text {code }}}{\mathrm{Pe}_{\text {code }} \exp \left(L c_{r k}\right)+1-\mathrm{Pe}_{\text {code }}},
$$

where $L c_{r k}$ and $\widetilde{L c_{r k}}$ are the $k$ th elements of $\mathbf{L} \mathbf{c}_{r}$ and $\tilde{\mathbf{L}} \mathbf{c}_{r}$, respectively.

Step 1. (Network decoding) At the $n$th iteration, the network decoder decodes $\mathbf{L} \mathbf{c}_{1}, \mathbf{L} \mathbf{c}_{2}, \tilde{\mathbf{L c}}_{r}$, with a priori information $\mathbf{I} \mathbf{A}_{1}^{n}$ and $\mathbf{I} \mathbf{A}_{2}^{n}$ to output the extrinsic information of coded bits $\mathbf{L} \mathbf{c}_{1}^{\mathrm{Net}(n)}$ and $\mathbf{L} \mathbf{c}_{2}^{\mathrm{Net}(n)}$. Let $L c_{1 k}, L c_{2 k}$, and $L c_{r k}$ be the $k$ th elements of $\mathbf{L} \mathbf{c}_{1}, \mathbf{L} \mathbf{c}_{2}$, and $\tilde{\mathbf{L} \mathbf{c}_{r}}$, respectively; $L c_{1 k}^{\mathrm{Net}(n)}$ and $L c_{2 k}^{\operatorname{Net}(n)}$ be the $k$ th element of $\mathbf{L} \mathbf{c}_{1}^{\operatorname{Net}(n)}$ and $\mathbf{L} \mathbf{c}_{2}^{\operatorname{Net}(n)}$, respectively; and $\mathrm{IA}_{1 k}^{n}$ and $\mathrm{IA}_{2 k}^{n}$ be the $k$ th elements of $\mathbf{I A}_{1}^{n}$ and $\mathbf{I A}_{2}^{n}$, respectively. Then

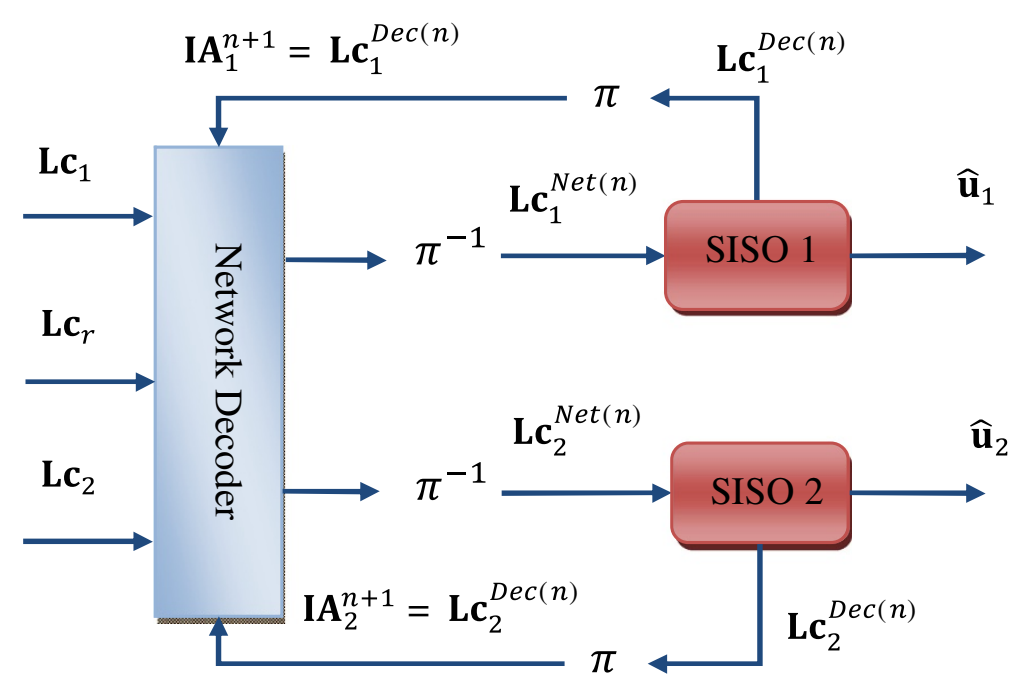

Figure 4 Diagram of the proposed JNCD algorithm 2. 


$$
\begin{aligned}
& \left.L c_{1 k}^{\mathrm{Net}(n)}\right)=L c_{1 k}+\log \frac{\exp \left(\widetilde{L c_{r k}}\right)+\exp \left(L c_{2 k}+\mathrm{IA}_{2 k}^{n}\right)}{1+\exp \left(\widetilde{L} c_{r k}+L c_{2 k}+\mathrm{IA}_{2 k}^{n}\right)} \\
& \left.L c_{2 k}^{\mathrm{Net}(n)}\right)=L c_{2 k}+\log \frac{\exp \left(\widetilde{L c_{r k}}\right)+\exp \left(L c_{1 k}+\mathrm{IA}_{1 k}^{n}\right)}{1+\exp \left(\widetilde{L} c_{r k}+L c_{1 k}+\mathrm{IA}_{1 k}^{n}\right)} .
\end{aligned}
$$

At the first iteration, $\mathbf{I} \mathbf{A}_{1}^{1}=\mathbf{I} \mathbf{A}_{2}^{1}=0$.

Step 2. (Channel decoding) The SISO decoder $j, j=1,2$, run the BCJR algorithm [25] as follows: Input: extrinsic information of coded bits $\mathbf{L} \mathbf{c}_{j}^{\mathrm{Net}(n)}$; the a priori extrinsic of information bits is equal to 0. Output: extrinsic information of coded bits $\mathbf{L} \mathbf{c}_{j}^{\operatorname{Dec}(n)}$.

Step 3. (Feedback) The extrinsic information of coded bits $\mathbf{L} \mathbf{c}_{1}^{\text {Dec }}, \mathbf{L c}_{2}^{\text {Dec }}$ is feedback to the network decoder as a priori (of coded bits) information for the next iteration: $\mathbf{I} \mathbf{A}_{j}^{n+1}=\mathbf{L c}_{j}^{\operatorname{Dec}(n)}, j=1,2$.

Step 4. Repeat from Step 1.

\section{Error probability estimation and quantization}

In order to apply the algorithms described in the previous section, the destination must estimate $\mathrm{Pe}_{\mathrm{bit}}=P\left[w^{r} \neq\right.$ $\left.u_{1} \oplus u_{2}\right]$ and $\mathrm{Pe}_{\text {code }}=P\left[c^{r} \neq c_{1} \oplus c_{2}\right]$. In this section, we compute these probabilities. Let $\operatorname{Pe}_{\mathrm{bit}}(j)=P\left[u_{j}^{r} \neq u_{j}\right]$ and $\operatorname{Pe}_{\text {code }}(j)=P\left[c_{j}^{r} \neq c_{j}\right], j=1,2$ be the decoding error probability of information bits and coded bits, respectively, of the link from source MSj to the relay. We assume, for simplicity, that the network-encoded information bits and network-encoded coded bits are independent (a reasonable assumption if interleavers are used at the relay).

The decoding error probability at the relay, $\mathrm{Pe}_{\mathrm{bit}}$, can be computed as follows:

$$
\begin{aligned}
\mathrm{Pe}_{\mathrm{bit}} & =P\left[w^{r} \neq u_{1} \oplus u_{2}\right] \\
& =\frac{1}{2} P\left[w^{r}=1 \mid u_{1} \oplus u_{2}=0\right]+\frac{1}{2} P\left[w^{r}\right. \\
& \left.=0 \mid u_{1} \oplus u_{2}=1\right] .
\end{aligned}
$$

The first factor can be computed as follows:

$$
\begin{aligned}
P\left[w^{r}=\right. & \left.1 \mid u_{1} \oplus u_{2}=0\right] \\
= & \frac{1}{2} P\left[w^{r}=1 \mid u_{1}=0, u_{2}=0\right]+\frac{1}{2} P\left[w^{r}=1 \mid u_{1}\right. \\
= & \left.1, u_{2}=1\right]=\frac{1}{2} P\left[u_{1}^{r}=0, u_{2}^{r}=1 \mid u_{1}=0, u_{2}\right. \\
= & 0]+\frac{1}{2} P\left[u_{1}^{r}=1, u_{2}^{r}=0 \mid u_{1}=0, u_{2}=0\right] \\
& +\frac{1}{2} P\left[u_{1}^{r}=0, u_{2}^{r}=1 \mid u_{1}=1, u_{2}=1\right]+\frac{1}{2} P\left[u_{1}^{r}\right. \\
= & \left.1, u_{2}^{r}=0 \mid u_{1}=1, u_{2}=1\right]=\frac{1}{2} P\left[u_{1}^{r}=0 \mid u_{1}\right.
\end{aligned}
$$

$$
\begin{aligned}
& =0] P\left[u_{2}^{r}=1 \mid u_{2}=0\right]+\frac{1}{2} P\left[u_{1}^{r}=1 \mid u_{1}=0\right] P\left[u_{2}^{r}\right. \\
& \left.=0 \mid u_{2}=0\right]+\frac{1}{2} P\left[u_{1}^{r}=0 \mid u_{1}=1\right] P\left[u_{2}^{r}=1 \mid u_{2}\right. \\
& =1]+\frac{1}{2} P\left[u_{1}^{r}=1 \mid u_{1}=1\right] P\left[u_{2}^{r}=0 \mid u_{2}=1\right] \\
& =\left(1-\operatorname{Pe}_{\mathrm{bit}}(1)\right) \operatorname{Pe}_{\mathrm{bit}}(2)+\left(1-\operatorname{Pe}_{\mathrm{bit}}(2)\right) \operatorname{Pe}_{\mathrm{bit}}(1)
\end{aligned}
$$

where the expression in (13) is given by the definition of XOR network coding.

Likewise, we have:

$$
\begin{aligned}
P\left[w^{r}=0 \mid u_{1} \oplus u_{2}=1\right]= & \left(1-\operatorname{Pe}_{\mathrm{bit}}(1)\right) \operatorname{Pe}_{\mathrm{bit}}(2) \\
& +\left(1-\operatorname{Pe}_{\mathrm{bit}}(2)\right) \operatorname{Pe}_{\mathrm{bit}}(1) .
\end{aligned}
$$

From (12) to (14) we have:

$$
\operatorname{Pe}_{\mathrm{bit}}=\operatorname{Pe}_{\mathrm{bit}}(1)+\operatorname{Pe}_{\mathrm{bit}}(2)-2 \mathrm{Pe}_{\mathrm{bit}}(1) \operatorname{Pe}_{\mathrm{bit}}(2) .
$$

The decoding error probability at the relay, $\mathrm{Pe}_{\text {code }}$, can be computed using similar steps as follows:

$$
\begin{aligned}
\text { Pe }_{\text {code }} & =P\left[c^{r} \neq c_{1} \oplus c_{2}\right] \\
& =\frac{1}{2} P\left[c^{r}=1 \mid c_{1} \oplus c_{2}=0\right]+\frac{1}{2} P\left[c^{r}\right. \\
& \left.=0 \mid c_{1} \oplus c_{2}=1\right] .
\end{aligned}
$$

The first factor in (16) can be computed in the same manner as in (13) as follows:

$$
\begin{aligned}
P\left[c^{r}=1 \mid c_{1} \oplus c_{2}=0\right]= & \left(1-\operatorname{Pe}_{\text {code }}(1)\right) \operatorname{Pe}_{\text {code }}(2) \\
& +\left(1-\operatorname{Pe}_{\text {code }}(2)\right) \operatorname{Pe}_{\text {code }}(1) .
\end{aligned}
$$

Likewise, we have:

$$
\begin{aligned}
P\left[c^{r}=0 \mid c_{1} \oplus c_{2}=1\right]= & \left(1-\operatorname{Pe}_{\text {code }}(1)\right) \operatorname{Pe}_{\text {code }}(2) \\
& +\left(1-\operatorname{Pe}_{\text {code }}(2)\right) \operatorname{Pe}_{\text {code }}(1) .
\end{aligned}
$$

From (16) to (18) we have:

$$
\operatorname{Pe}_{\text {code }}=\operatorname{Pe}_{\text {code }}(1)+\operatorname{Pe}_{\text {code }}(2)-2 \operatorname{Pe}_{\text {code }}(1) \operatorname{Pe}_{\text {code }}(2) \text {. }
$$

In the next subsections, $\operatorname{Pe}_{\mathrm{bit}}(j)=P\left[u_{j}^{r} \neq u_{j}\right]$ and $\operatorname{Pe}_{\text {code }}(j)=P\left[c_{j}^{r} \neq c_{j}\right], j=1,2$ are computed for different fading scenarios. In our analysis, we assume Gray mapping. Also, the nearest neighbor approximation is used. This corresponds to the assumptions that if an error occurs, then the transmitted symbol can only be one of the symbols closest to the estimated one. Therefore, due to Gray mapping, one symbol error causes a single coded bit error. As illustrative examples, three cases are considered: $F=1, F=4$, and fully interleaved fading. $\mathrm{Pe}_{\text {bit }}^{F 1, F 4, F u l l}\left(\mathrm{Pe}_{\text {code }}^{F 1, F 4, F u l l}\right)$ denote the decoding error probability of information bits (coded bits) for each case study, 
respectively. For simplicity, we focus our attention on 16-QAM modulation, as used in our numerical examples.

\subsection{Error estimation with perfect CSI: computation of $\mathrm{Pe}^{F 1, F 4 \text {,Full }}$}

\subsubsection{Block Rayleigh fading $F=1$}

In this case, the channels $\mathrm{MSj}-\mathrm{R} j=1,2$ are Gaussiandistributed conditioned on $h_{j}$. The symbol error probability of the $M S_{j}-R$ link for M-QAM modulation is ([27], Equation 5 .2.79):

$$
\operatorname{Psym}_{M}(j)=1-\left(1-\operatorname{Psym}_{\sqrt{M}}\right)^{2},
$$

with

$$
\operatorname{Psym}_{\sqrt{M}}=2\left(1-\frac{1}{\sqrt{M}}\right) Q\left(\sqrt{\frac{3}{M-1} \frac{\left|h_{j}\right|^{2} \mathrm{Es}}{\sigma_{n}^{2}}}\right),
$$

where $\mathrm{Q}($.$) denotes the \mathrm{Q}$-function, $h_{j}$ is the channel gain, and Es is the symbol energy. Because each symbol error causes one coded bit error (Gray mapping and nearest neighbor approximation), then the error probability of coded bits of, for example, 16-QAM modulation can be estimated as follows:

$$
\begin{aligned}
\operatorname{Pe}_{\text {code }}^{F 1}(j) & =\operatorname{Psym}_{M}(j) / \sqrt{M} \\
& \approx \frac{3}{8} \operatorname{erfc}\left(\sqrt{\frac{\left|h_{j}\right|^{2} \mathrm{Es}}{10 \sigma_{n}^{2}}}\right),
\end{aligned}
$$

where $\operatorname{erfc}(\cdot)$ is related to the $\mathrm{Q}$-function.

\subsubsection{Block Rayleigh fading $F=4$}

Let $h_{j}=\left[h_{h 1}, h_{j 2}, h_{j 3}, h_{j 4}\right]$ be the channel gain vector of link MSj-R $j=1,2$. Because the components of $h_{j}$ are independent, the error probability $\operatorname{Pe}_{\text {code }}^{F 4}(j)$ of 16-QAM modulation is estimated as the average over the vector $h_{j}$ :

$$
\operatorname{Pe}_{\text {code }}^{F 4}(j)=\frac{1}{4} \sum_{k=1}^{4}\left[\frac{3}{8} \operatorname{erfc}\left(\sqrt{\frac{\left|h_{j k}\right|^{2} \mathrm{Es}}{10 \sigma_{n}^{2}}}\right)\right] .
$$

\subsubsection{Fully-interleaved Rayleigh fading}

The error probability of coded bits of link MSj-R over fully interleaved Rayleigh fading channel is computed by integrating over the distribution of the channel gains. Let $h_{j}$ be channel gain, then $\gamma=\left|h_{j}\right|^{2}$ is exponentially distributed with mean equal to $E\left[\left|h_{j}\right|^{2}\right]=1$. Therefore, the symbol error rate of link MSj-R with M-QAM modulation is:

$$
\begin{aligned}
\operatorname{Psym}_{M}^{\text {Full }}(j) & =\int_{0}^{\infty} \frac{\sqrt{(} M)-1}{M} e^{-\gamma} \operatorname{erfc}\left(\sqrt{\frac{3 \mathrm{Es}}{2(M-1) \sigma_{n}^{2}} \gamma}\right) d \gamma \\
& =2\left(1-\frac{1}{\sqrt{M}}\right)\left(1-\sqrt{\frac{3 \mathrm{Es}}{3 \mathrm{Es}+2(M-1) \sigma_{n}^{2}}}\right), j=1,2 .
\end{aligned}
$$

The coded bit error probability of 16-QAM modulation is thus equal to:

$$
\begin{aligned}
\operatorname{Pe}_{\text {code }}^{\text {Full }}(j) & =\operatorname{Psym}^{\text {Full }}{ }_{M}(j) / \sqrt{M} \\
& =\frac{3}{8}\left(1-\sqrt{\frac{\mathrm{Es}}{\mathrm{Es}+10 \sigma_{n}^{2}}}\right) .
\end{aligned}
$$

\subsection{Error estimation with perfect CSI: computation of $\mathrm{Pe}_{\text {bit }}^{F 1, F 4 \text {,Full }}$}

The information bit error probability of convolutional codes conditioned on the channel vector can be computed as follows ([28], Equation 3.175):

$$
\mathrm{Pe}_{\mathrm{bit}} \approx \sum_{d=d_{f}}^{\infty} \beta(d) P_{c}(d)
$$

where $d_{f}$ is the minimum distance, $\beta(d)$ is the distance spectrum of the convolutional code, and $P_{c}(d)$ is the probability of choosing a wrong path in the trellis with distance $d$ from the correct path (usually the all-zero path). $P_{c}(d)$ depends on the channel gains and is computed as follows.

\subsubsection{Block Rayleigh fading channel with $F=1$}

Let $P_{c}^{j}(d)$ be the conditional pairwise error probability related to the MSj-R channel. Since Gray mapping is used and the nearest neighbor approximation is assumed, each symbol error only causes one error on the coded bits. In addition, the coded bits are interleaved before being mapped into the constellation. Thus, the conditional pairwise error probability of the MSj-R link is

$$
P_{c}^{j}(d)=\frac{3}{4} \operatorname{erfc}\left(\sqrt{\frac{d\left|h_{j}\right|^{2} \mathrm{Es}}{10 \sigma_{n}^{2}}}\right) .
$$

By substituting (25) in (24), the bit error probability of the $\mathrm{MSj}-\mathrm{R}$ link is estimated as follows:

$$
\mathrm{Pe}_{\mathrm{bit}}^{F 1}(j) \approx \frac{3}{4} \sum_{d=d_{f}}^{\infty} \beta(d) \operatorname{erfc}\left(\sqrt{\frac{d\left|h_{j}\right|^{2} \mathrm{Es}}{10 \sigma_{n}^{2}}}\right) .
$$

In our simulation results, $d_{f}=6$ for the RSC code [1 15/13] and only two values of $d$ are used.

\subsubsection{Block Rayleigh fading channel with $F=4$}

The conditional pairwise error probability $P_{c}^{j}(d)$ on the MSj-R link depends on the channel vector $\mathbf{h}_{j}=\left\{h_{j f}\right\}_{f=1}^{4}$ and the distribution of the weight $d$ over the $F$ blocks. Denote by $d_{f}$ the number of weights in block $f, f=$ $1,2, \ldots F$ subject to $0 \leq d_{f} \leq d$ and $\sum_{f=1}^{F} d_{f}=d$. Then the distribution of the weight $d$ over the $F$ blocks is given by the weight pattern $\mathbf{D}=\left\{d_{f}\right\}_{f=1}^{F}$. The conditional pairwise error probability is estimated by averaging over all the weight patterns $\mathbf{D}$ : 


$$
P_{c}^{j}(d)=\sum_{\mathbf{D}} P_{c}^{j}(d \mid \mathbf{D}) p(\mathbf{D})
$$

where the pairwise error probability given the weight pattern $\mathbf{D}$ is computed as [22]:

$$
P_{c}^{j}(d \mid \mathbf{D})=\frac{3}{4} \operatorname{erfc}\left(\sqrt{\frac{E s}{10 \sigma_{n}^{2}} \sum_{f=1}^{F} d_{f}\left|h_{j f}\right|^{2}}\right),
$$

and the distribution of the pattern $\mathbf{D}$ is computed using combinatorial analysis:

$$
p(\mathbf{D})=\frac{\prod_{f=1}^{F} \mathcal{C}_{d_{f}}^{m}}{\mathcal{C}_{d}^{N_{s}}}
$$

where $\mathcal{C}_{k}^{n}$ denotes a combination of $k$ elements of a set of $n$ elements; $N_{s}=N / \log _{2}(M)$ is the length of a signal; $m=$ $N_{s} / F$ is the block's length.

From (24), (27), and (28), the error probability reduces to:

$$
\mathrm{Pe}_{\mathrm{bit}}^{F 4}(j) \approx \frac{3}{4} \sum_{d=d_{f}}^{\infty} \beta(d) \sum_{\mathbf{D}} \operatorname{erfc}\left(\sqrt{\frac{\mathrm{Es}}{10 \sigma_{n}^{2}} \sum_{f=1}^{F} d_{f}\left|h_{j f}\right|^{2}}\right) p(\mathbf{D}) .
$$

\subsubsection{Fully interleaved Rayleigh fading}

The error probability of coded bits does not depend on the instantaneous channel gain but on the noise variance only. The pairwise error probability in fully interleaved fading channel can be obtained by integrating over the distribution of channel gains. Using [28], we get:

$$
\mathrm{Pe}_{\mathrm{bit}}^{\text {Full }}(j)=\frac{3 \beta\left(d_{f}\right)}{2} \mathcal{C}_{d_{f}}^{2 d_{f}-1}\left(\frac{2 \mathrm{Es}}{5 \sigma_{n}^{2}}\right)^{-d_{f}},
$$

where $\mathcal{C}_{m}^{n}=\frac{n !}{m !(n-m) !}$ denotes the binomial coefficients.

\subsection{Error estimation with imperfect perfect CSI}

In the imperfect CSI case, we consider $F=1$ and $F=4$. The error probabilities at the relay can be computed as in the perfect CSI case, except that the estimated channel gain $\hat{h}_{j}, j=1,2$ is used instead of correct one $h_{j}$.

\subsection{Error quantization}

To inform the destination about the decoding error probability at the relay, the relay quantizes and sends it to the destination. Let $\bar{v}$ be a $q$-bit quantized value of $v$ using uniform quantization function $Q_{q}($.$) :$

$$
\begin{aligned}
\bar{v}=Q_{q}(v) & =\frac{k M_{v}}{2^{q}}, \text { if } \frac{(k-1) M_{v}}{2^{q+1}} \leq v<\frac{(k+1) M_{v}}{2^{q+1}}, k \\
& =1,2, \ldots, 2^{q}
\end{aligned}
$$

where $M_{v}=\max (v)-\min (v)$. The quantization error by $Q_{q}\left(\right.$.) is given by $\varepsilon_{q}=M_{v} / 2^{q+1}$. The quantized $\bar{v}$ is transmitted over fading plus Gaussian noise to the destination. At the end of the channel estimation phase, the destination recovers the decoding error probability at the relay from the noisy version of the transmitted quantized signal.

\section{Numerical results: perfect CSI case}

In this section, we study the performance of the proposed JNCD algorithms in various fading scenarios. For this perfect CSI case, assume that the receivers have perfect knowledge of the one-hop links CSI. In addition, the destination is assumed to have full knowledge of the decoding error probability at the relay, $\mathrm{Pe}_{\mathrm{bit}}$ and $\mathrm{Pe}_{\text {code }}$, which are estimated as described in the previous sections. We assume a symmetric network topology in which the distance from the sources to the destination is the same. The relay is located between sources and destination. The path loss has been chosen equal to 3.5 [29]. Unless stated for specific cases, the recursive convolutional code (RSC) [1 $15 / 3$ ] with rate $1 / 2$ is used. 16-QAM is used as the modulation scheme. The number of iterations used to obtain our results is four since we have observed that the algorithms converge to the best performance in four iterations.

Both DF and DMF relaying protocols are studied. In particular, three schemes are studied: (1) DF relaying with the proposed algorithm 1, named DF Algo 1 in the figure; (2) DF relaying with the proposed algorithm 2, named $D F$ Algo 2 in the figure; (3) DMF relaying with the proposed algorithm 2, named DMF Algo 2 in the figure. We note that algorithm 1 cannot be used with the DMF protocol because it performs network coding on the information bits. We also compare our algorithms with [18], which is denoted by Ref. [Yune] in the figure. In addition, we denote by Blind the scenario where the receiver has no information about the decoding error probability at the relay (it assumes perfect decoding at the relay) and by Non Cooperation the conventional point-to-point communication scenario.

\subsection{Effects of iterations}

Figure $5 \mathrm{a}$ shows the simulation results for fully interleaved fading channels. It is shown in the figure that (1) iterative network/channel decoding significantly improves the performance of both proposed algorithms using the DF protocol. More specifically, with four iterations, compared with separate decoding (one iteration), the proposed algorithm 1 gains $4 \mathrm{~dB}$ at a BER equal to $10^{-3}$. On the other hand, the proposed algorithm 2 gains $5 \mathrm{~dB}$. If the DMF protocol is considered, the proposed algorithm 2 with four iterations gains about $2 \mathrm{~dB}$ at a BER equal to $10^{-3}$ compared with one iteration decoding. (2) If the DF protocol is considered, the algorithm 2 is about 
a)

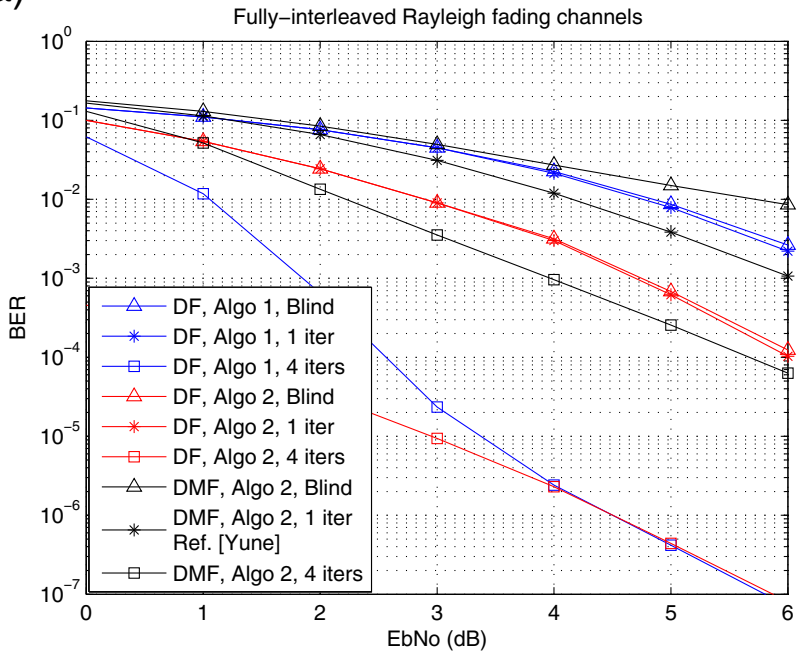

b)

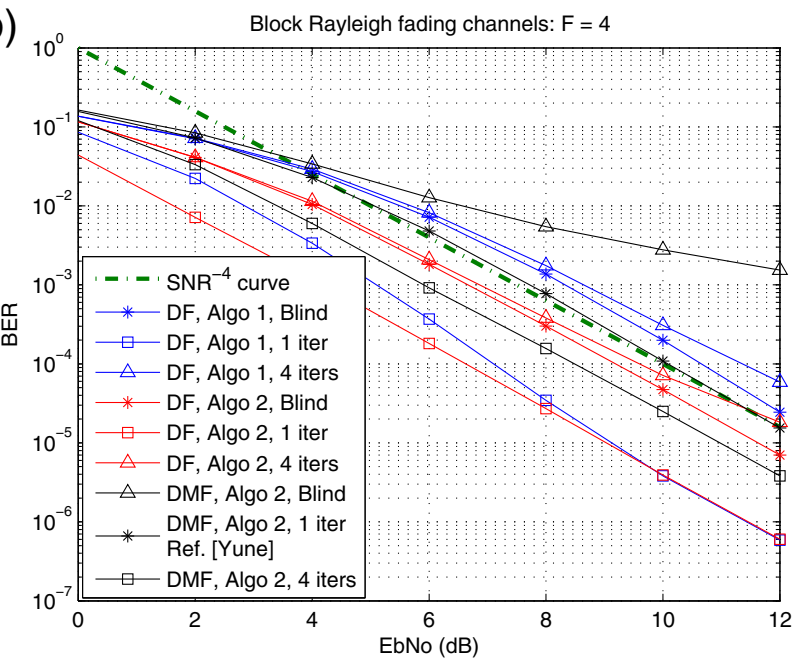

c)

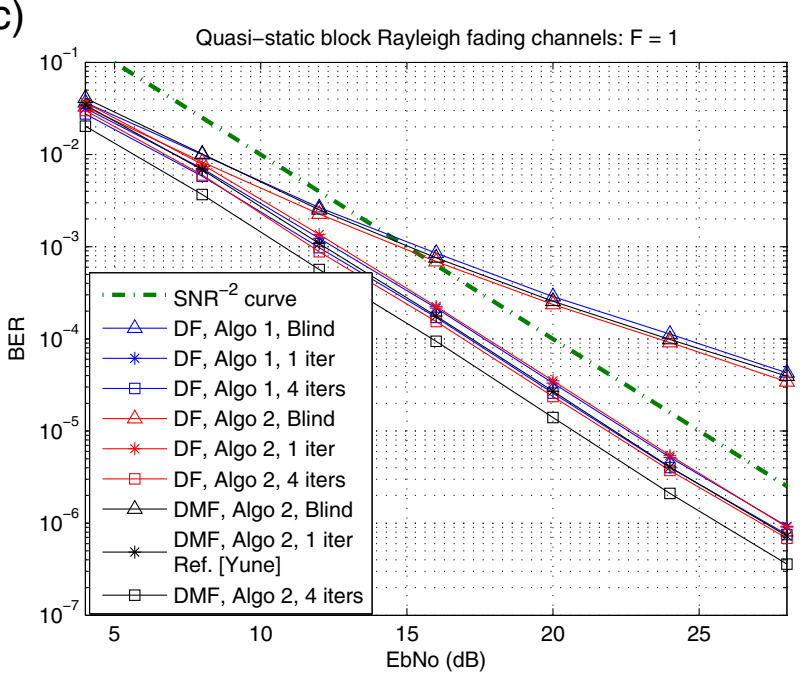

Figure 5 Performance of the proposed JNCD algorithms 1 and 2 in different Rayleigh fading scenarios with full CSI. (a) fully interleaved fading channel, (b) block fading channel $F=4$, (c) block fading channel $F=1$. 
$2 \mathrm{~dB}$ better than the algorithm 1 after one iteration. After four iterations, the two algorithms tend to have almost the same performance as SNR increases. (3) Compared to [18], the two proposed algorithms with four iterations and DF protocol significantly outperform.

Figure $5 \mathrm{~b}$ shows the simulation results for block fading channel with $F=4$. In addition, the theoretical curve $\mathrm{SNR}^{-4}$ is plotted to provide some information about the achievable diversity order: (1) iterative decoding improves performance for both DF and DMF relaying. With four iterations, DF relaying with the proposed algorithm 1 gains about $3 \mathrm{~dB}$ and with the proposed algorithm 2 , it gains about $2 \mathrm{~dB}$ at a BER equal to $10^{-4}$ compared to the single iteration case. DMF relaying with algorithm 2 gains about $1 \mathrm{~dB}$. (2) Compared to [18], after four iterations, algorithm 1 and algorithm 2 with DF relaying both gain about $3 \mathrm{~dB}$ at a BER equal to $10^{-4}$. With DMF relaying, the algorithm 2 with four iterations gains about $1 \mathrm{~dB}$ at a BER equal to $10^{-4}$. (3) For both DF and DMF relaying, the receiver loses the diversity order if it has no information about the decoding error probability.

We note that in Figure 5b, the proposed algorithms have diversity order equal to 4 if $F=4$. The reason is that the diversity gain $d^{H}$ of a convolutional code in a $F$-block fading channel with $M$-QAM modulation is upper bound by [22]:

$$
d^{H} \leq\left\lfloor F\left(1-\frac{R}{M}\right\rfloor+1,\right.
$$

where $\lfloor x\rfloor$ denotes the largest integer no greater than $x, R$ is the code rate in bits/symbol. In our setup, we have $R=2$ bits/symbol and 16-QAM. Thus, we get $d^{H} \leq 3$. It is shown from the simulation that the actual diversity order of the code [1 15/13] is 2 in the SNR range of interest. Therefore, it is reasonable that in the MARC, the relay provides a better diversity gain.

Figure $5 \mathrm{c}$ shows simulation results for the quasi-static fading channel with $F=1$. In addition, the theoretical curve $\mathrm{SNR}^{-2}$ is plotted as a diversity reference. It is shown in the figure that (1) if the receiver is not informed about the decoding error probability at the relay, the performance is dramatically decreased and it loses diversity order; (2) iterative decoding brings a little gain in both algorithms. Algorithm 2 with four iterations gains about $0.8 \mathrm{~dB}$ over the one-iteration case, while algorithm 1 with four iterations gains about $0.5 \mathrm{~dB}$ over the one-iteration case; and (3) DMF with algorithm 2 is slightly better than DF with both algorithms.

Consider three fading channels, algorithm 2 is recommended because of its low complexity compared with algorithm 1. With quasi-static block fading scenario, DMF relaying is recommended. In this case, channel decoding at the relay does not improve the system performance. In general $F$-block fading channels, DF relaying should be used.

\subsection{Effects of location of the relay}

Figure 6 shows the effects of relay's position on the performance of the proposed algorithms in $F=4$ block Rayleigh fading channels. The horizonal axis presents the normalized source-relay distance when source-destination distance is 1 . The system operates at EbNo $=6 \mathrm{~dB}$. It is shown

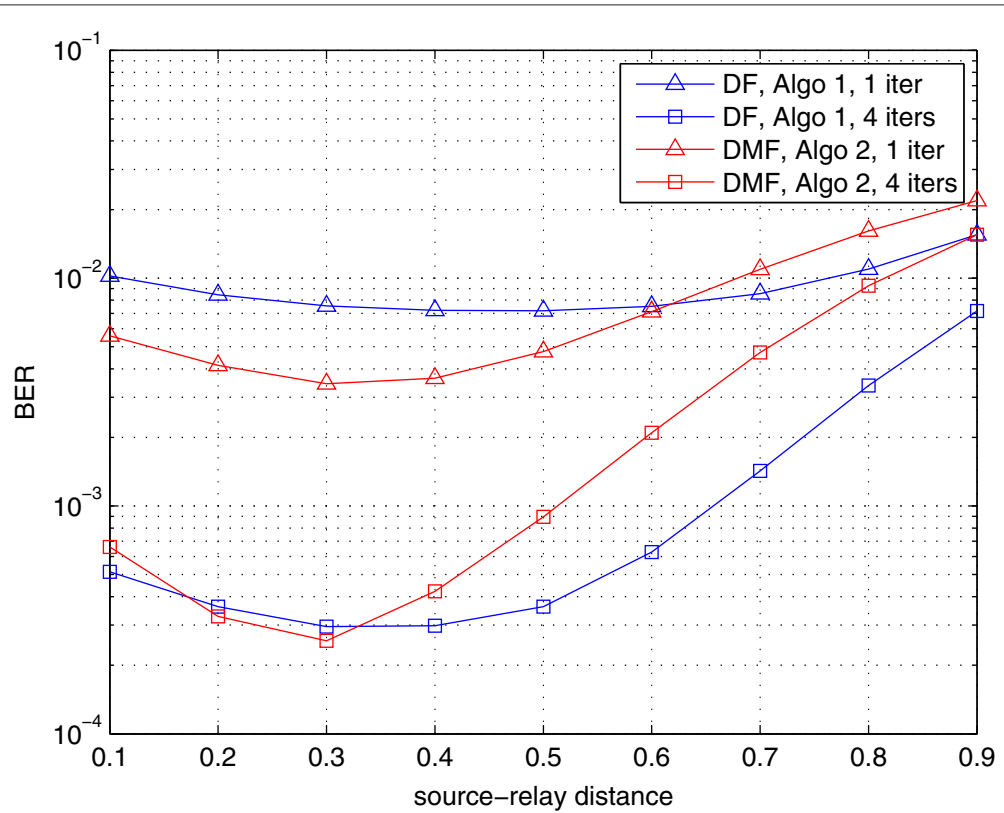

Figure 6 Effects of relay's location on performance of proposed JNCD algorithms under $F=4$ block Rayleigh fading channels with Full-Pe. 
that the system performance is degraded when the relay move close to the destination. It is because in this case, the possible errors at the relay are so high. The system achieves the best performance when the relay is close to the source. In addition, the optimal location for DMF is closer to the source than that for DF. Since DMF does not perform channel decoding, it needs the source-relay links to be good enough for effective cooperation.

\subsection{Effects of the channel code}

Figure 7 presents the performance of the proposed algorithm 1 with DF in $F=4$ block Rayleigh fading scenario and the relay locates at the middle between the sources and the relay. The destination has full knowledge of the decoding error at the relay. Two channel codes are compared. Code 1: RSC [1 15/13] with rate $1 / 2$ and Code 2 : RSC [1 15/13 17/13] with rate $1 / 3$. Obviously, the Code 2 with higher error-correction capability provides better performance than the Code 1 . In addition, the MARC with Code 2 achieves diversity order 6 while the MARC with Code 1 achieves diversity order 4 in the SNR region of interest. This is because in $F=4$ block fading, the Code 1 can only achieve diversity order 2 while the Code 2 provides diversity order 3 .

\section{Numerical results: imperfect CSI case}

This section evaluates the impact of imperfect CSI and quantization error on the performance of the proposed algorithms. The two case studies with $F=1$ and $F=4$ are investigated. The relay is located at the middle between the sources and the destination. The RSC [1 15/13] is chosen as in the previous section and 16-QAM is used. The ML estimator is used for channel estimation. Because the performance of algorithm 2 with DF and DMF relaying is almost the same, we only study algorithm 2 with DMF relaying in this section. Then, in this section, algorithm 1 is linked to DF relaying and algorithm 2 is linked to DMF relaying. In the figures, Full CSI denotes the case when the receivers (relay and destination) have perfect channel state information of the one-hop links. On the other hand, Full Pe denotes the case when the destination has full knowledge of the decoding error probability at the relay.

Intensive simulations show that iterative decoding only provides coding gain if the receivers have enough accurate CSI and possible decoding errors. However, concerning realistic imperfect CSI systems where the total number of overhead symbols is not too large, we focus on only separate decoding (one iteration) for block fadings ( $F=1$ and $F=4$ ) in what follows.

\subsection{Effects of pilot length}

Figure 8 shows the effect of pilot length assuming FullPe for both quasi-static and $F=4$ block fading channels. Both algorithms have the same performance trend as a function of number of pilot symbols. It is shown that pilot length only affects the coding gain, and it does not change the diversity order of the system in the SNR range of interest. In addition, 16-symbol pilot curve is about 1 - $\mathrm{dB}$ worse than the full CSI curve (at a BER equal to $10^{-4}$ ).

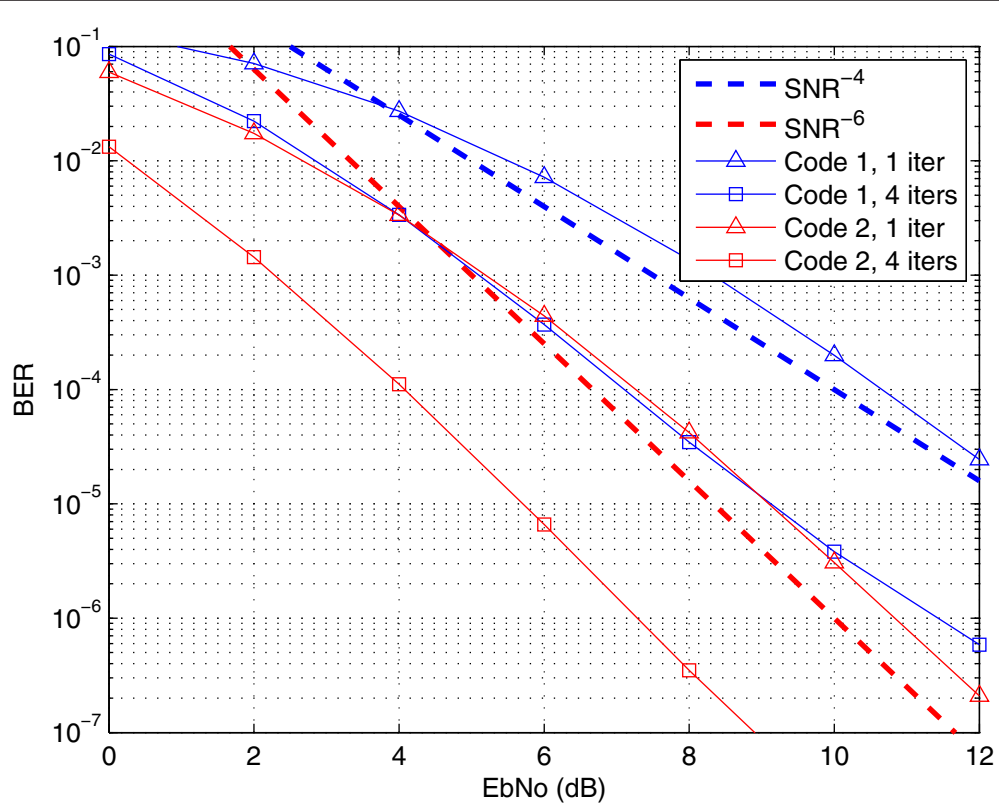

Figure 7 Performance of proposed JNCD algorithm 1 with difference channel codes in $F=4$ block fading scenario with DF relaying. 


\section{a)}

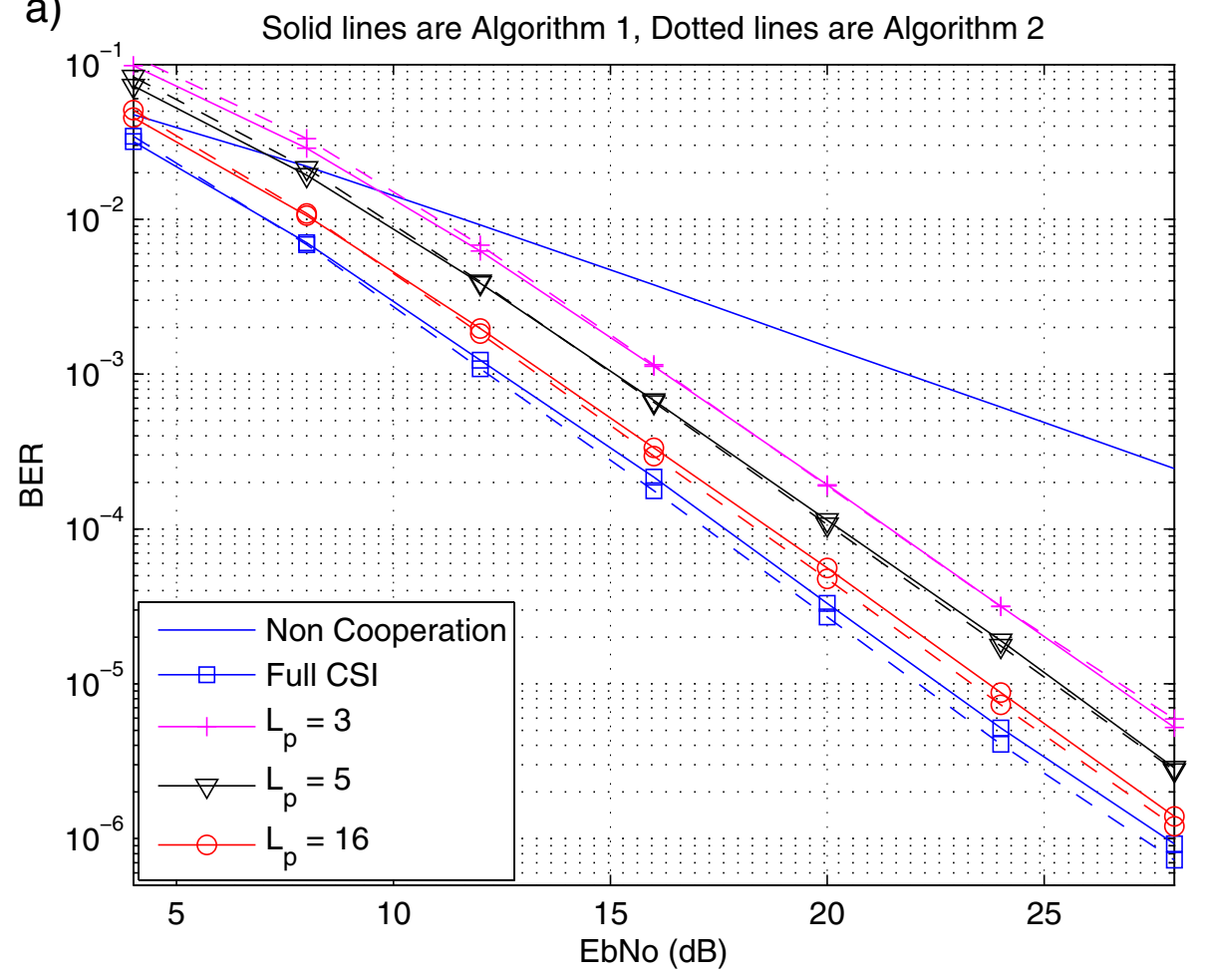

b) $10^{0} \quad$ Solid lines are Algorithm 1, Dotted lines are Algorithm 2

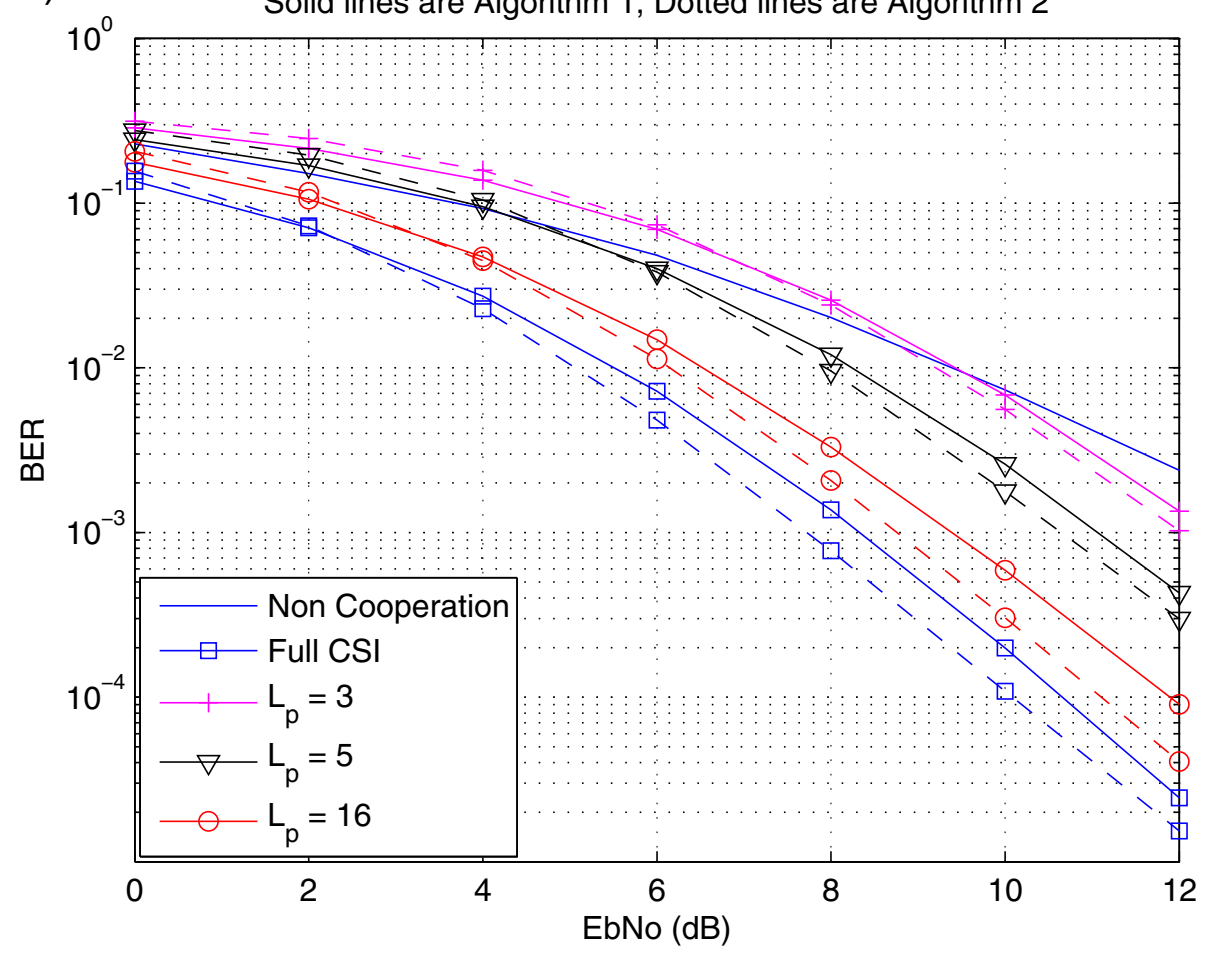

Figure 8 Effects of pilots on performance of proposed algorithms: (a) $F=1$ block fading channel, (b) $F=4$ block fading channel. 


\subsection{Effects of quantization}

Figure 9 illustrates the effect of quantization by assuming five pilot symbols and $F=1$. We note that the total overhead symbols (pilot symbols and quantization symbols) are five, the pilot symbols on relay-destination link is less than five. It is observed that if the BER at the relay is not taken into account by the decoder at destination, it loses both coding gain and diversity order. Furthermore, the quantization level, $L_{q}$, affects both performance and diversity order. In both algorithms, $L_{q}=4$ bits quantization ( $L_{p}=1$ symbol for relay-destination link) can achieve almost same performance as the full Pe case. It implies that the system BER is more sensitive to the quantization accuracy than the pilot length.

Figure 10 shows the effect of quantization with perfect CSI and $F=4$. Similar conclusions as above are derived. The quantization level affects both diversity order and coding gain. The quantization level has small effect to the algorithm 1 and it achieves almost same performance as full Pe case in the observing SNR range. This

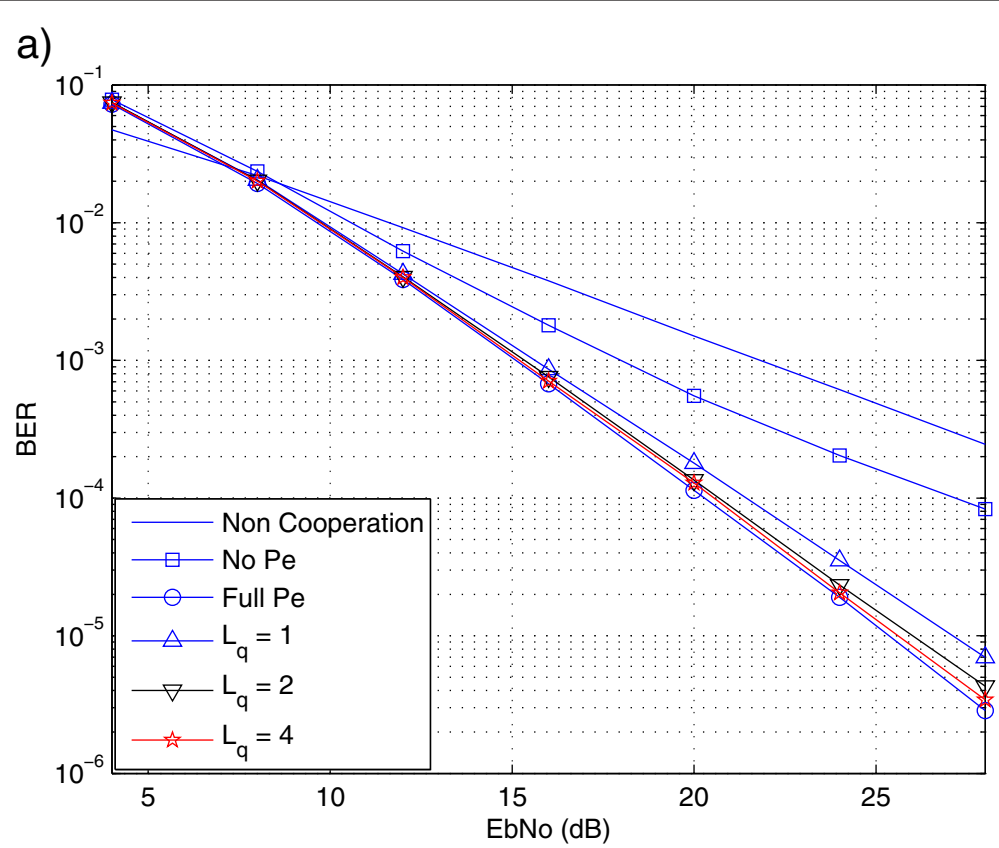

b)

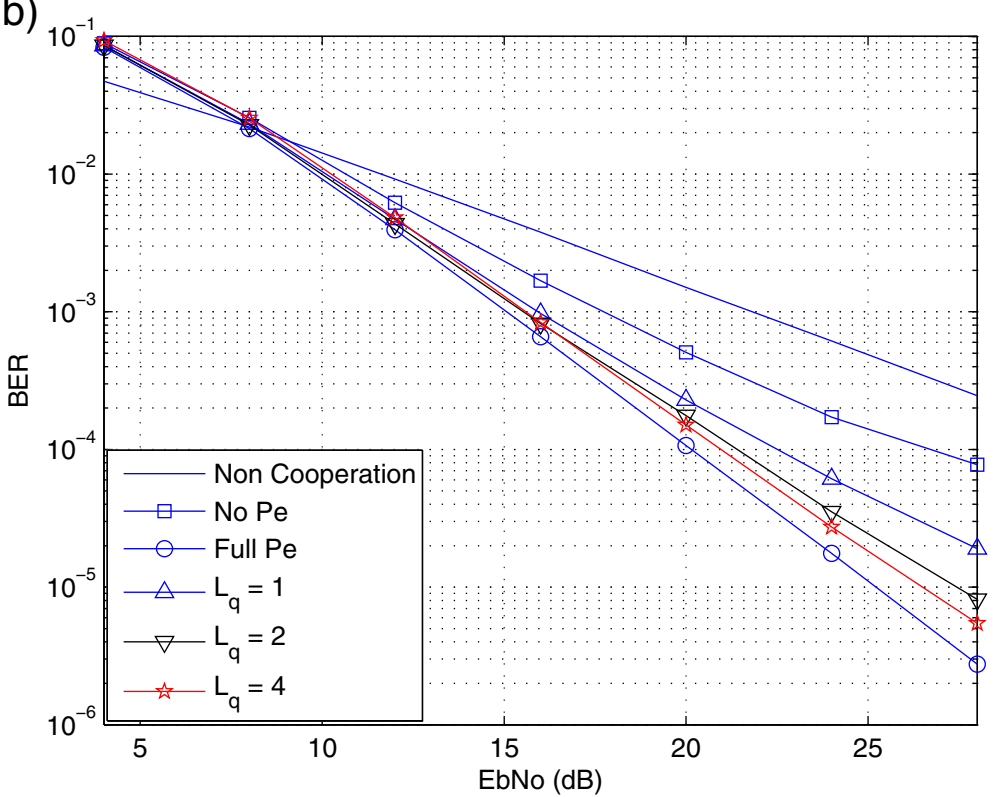

Figure 9 Effects of quantization level $L_{q}$ on performance of proposed algorithms in block fading $F=1$ with $L_{p}=5$ pilot symbols. 
a)

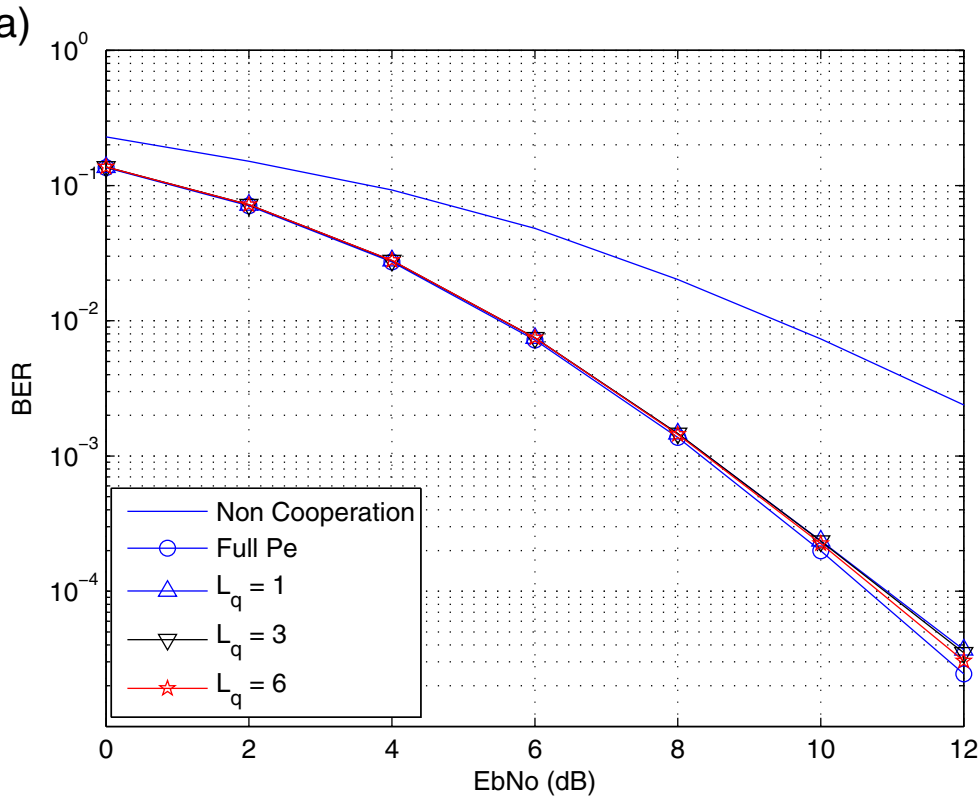

b)

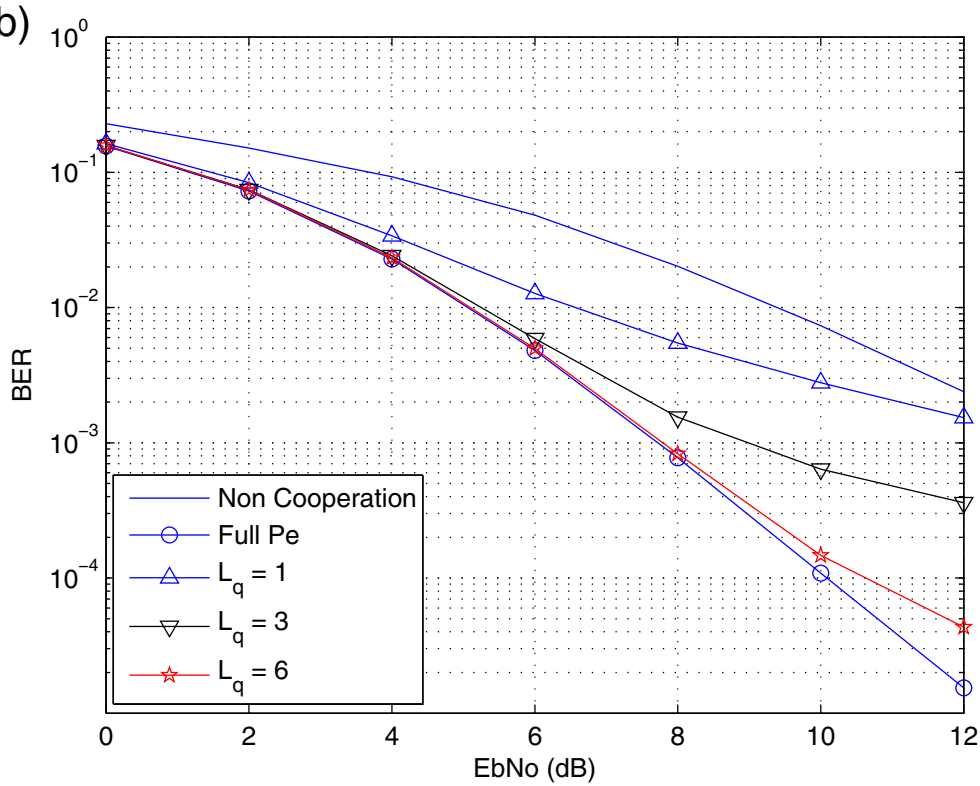

Figure 10 Effects of quantization level $L_{q}$ on performance of proposed algorithms in block fading $F=4$ with perfect CSI. a) The proposed algorithm 1. b) The proposed algorithm 2.

is because the algorithm 1 performs channel decoding at the relay and the possible decoding errors are negligible in this situation. Unlike algorithm 1, the performance of the algorithm 2 significantly degrades with quantized errors, even when $L_{q}=6$ bits are used. This is because of two following reasons: (1) the algorithm 2 employs DMF relaying, which results in high errors at the relay and (2) the decoding errors at the relay is computed as the average over four channels, which is not accurate for individual independent channels.
In conclusion, (1) if $F=1$, algorithm 2 has the same performance as algorithm 1 and it is less complex than algorithm 1 . However, if $F=4$, algorithm 1 outperforms algorithm 2. (2) If the destination is aware enough of the decoding error probability at the relay, pilot length does not change that diversity order of the system. (3) The number of quantization bits for reporting decoding errors from the relay affects both coding gain and diversity order. (4) Six-bit quantization is enough in most analyzed scenarios. 


\section{Conclusion}

In this paper, we have studied the performance of the multiple access relay channel with binary Network Coding and JNCD at the destination in practical situations. Decode and Forward and Demodulate and Forward relaying strategies are investigated. Our results show that iterative Joint Network and Channel Decoding provides better performance than separate network channel decoding only if the destination has enough CSI and knowledge of the decoding error probability at the relay. This gain increases with the number of fading blocks per codeword. It is also shown that the number of pilot symbols mostly affects the coding gain of the system with a negligible impact on the diversity order, at least for the SNR range of interest. Finally, it is shown that CSI quantization errors affect both coding gain and diversity order. In general, representing the BER at the relay using 6-bit quantization is sufficient for both DMF relaying and DF relaying. The proposed iterative decoding algorithms can be easily extended to frequency selective fading scenarios, e.g., OFDM systems.

\section{Competing interests}

The authors declare that they have no competing interests.

\section{Acknowledgements}

The research work is supported in part by the European Commission under the auspices of the FP7-PEOPLE MITN-CROSS FIRE project (Grant 317126) and in part by the European Commission in the framework of the FP7 Network of Excellence in Wireless COMmunications NEWCOM\# (Grant agreement no. 318306).

\section{Received: 8 April 2013 Accepted: 23 October 2013}

Published: 11 November 2013

\section{References}

1. A Sendonaris, E Erkip, B Aazhang, User cooperation diversity - Part I: system description. IEEE Trans. Commun. 51(11), 1927-1938 (2003)

2. JN Laneman, DNC Tse, GW Wornell, Cooperative diversity in wireless networks: Efficient protocols and outage behavior. Inform. Theory, IEEE Trans. 50(12), 3062-3080 (2004)

3. R Ahlswede, C Ning, SYR Li, RW Yeung, Network information flow. Inform Theory, IEEE Trans. 46(4), 1204-1216 (2000)

4. M Renzo, L Iwaza, M Kieffer, P Duhamel, K Agha, Robust wireless network coding-an overview, in Lecture Notes of the Institute for Computer Sciences. Social Informatics and Telecommunications Engineering, Vol. 45 (Springer, Berlin Heidelberg, 2010), pp. 685-698

5. Y Sichao, R Koetter, Network coding over a noisy relay : a belief propagation approach. in the IEEE International Symposium on Information Theory, ISIT 2007. Nice, 24-29 June 2007

6. DH Woldegebreal, $\mathrm{H}$ Karl, Multiple-access relay channel with network coding and non-ideal source-relay channels in the 4th International Symposium on Wireless Communication Systems, ISWCS 2007. Trondheim, 17-19 October 2007

7. C Hausl, P Dupraz, Joint network-channel coding for the multiple-access relay channel in the $20063 \mathrm{rd}$ Annual IEEE Communications Society on Sensor and Ad Hoc Communications and Networks, SECON '06. Reston, 25-28 September Vol. 3 (IEEE Piscataway 2006), pp. 817-822

8. ZA Polgar, MP Stef, V Bota, Network and channel coded cooperation algorithms for cellular networks in the IEEE 69th Vehicular Technology Conference 2009, VTC Spring. Barcelona, 26-29 April, 2009

9. L Yonghui, B Vucetic, TF Wong, M Dohler, Distributed turbo coding with soft information relaying in multihop relay networks. Select. Areas Commun., IEEE J. 24(11), 2040-2050 (2006)
10. P Weitkemper, D Wuebben, V Kuehn, KD Kammeyer, Soft information relaying for wireless networks with error- prone source-relay link, in the 7th International ITG Conference on Source and Channel Coding (SCC). Ulm 14-16 January 2008

11. HH Sneesens, L Vandendorpe, Soft decode and forward improves cooperative communications, in the 1st IEEE International Workshop on Computational Advances in Multi-Sensor Adaptive Processing. Puerto Vallarta, 13-15 December, 2005

12. Al-G Habian, A Ghrayeb, M Hasna, Controlling error propagation in network-coded cooperative wireless systems, in the IEEE International Conference on Communications, ICC'09. Dresden, 14-16 June 2009, pp. 1-6

13. N Sinh Le Hong, A Ghrayeb, Al-G Habian, M Hasna, Mitigating error propagation in two-way relay channels with network coding. Wireless Commun. IEEE Trans. 9(11), 3380-3390 (2010)

14. A Bletsas, A Khisti, DP Reed, A Lippman, A simple cooperative diversity method based on network path selection. IEEE J. Select Areas Commun. 24(3), 659-672 (2006)

15. X Ming, T Aulin, Optimal decoding and performance analysis of a noisy channel network with network coding. Commun, IEEE Trans. 57(5), 1402-1412 (2009)

16. HH Sneessens, J Louveaux, L Vandendorpe, Turbo-coded decode-and-forward strategy resilient to relay errors, in the IEEE International Conference on Acoustics, Speech and Signal Processing, ICASSP 2008. Las Vegas, 30 March - 4 April 2008

17. K Lee, L Hanzo, MIMO-assisted hard versus soft decoding-and-forwarding for network coding aided relaying systems. Wireless Commun. IEEE Trans. 8, 376-385 (2009)

18. TYune, D Kim, G Im, Opportunistic network-coded cooperative transmission with demodulate-and-forward protocol in wireless channels. Commun, IEEE Trans. PP(99), 1-5 (2011)

19. TT Kim, G Caire, M Skoglund, Decode-and-forward relaying with quantized channel state feedback: an outage exponent analysis. Inform. Theory, IEEE Trans. 54(10), 4548-4564 (2008)

20. D Zhiguo, KK Leung, Impact of imperfect channel state information on bi-directional communications with relay selection. Signal Process IEEE Trans. 59(11), 5657-5662 (2011)

21. B Nazer, M Gastpar, Compute-and-forward: Harnessing interference with structured codes, in IEEE International Symposium on Information Theory, 2008. ISIT 2008. Toronto, 6-11 July 2008

22. R Knopp, PA Humblet, On coding for block fading channels. Inform. Theory IEEE Trans. 46, 189-205 (2000)

23. JK Cavers, An analysis of pilot symbol assisted modulation for Rayleigh fading channels [mobile radio]. Vehicular Technol. IEEE Trans. 40(4), 686-693 (1991)

24. T Lang, BM Sadler, M Dong, Pilot-assisted wireless transmissions: general model, design criteria, and signal processing. Signal Process Mag. IEEE. 21(6), 12-25 (2004)

25. L Bahl, J Cocke, F Jelinek, J Raviv, Optimal decoding of linear codes for minimizing symbol error rate (Corresp.) Inform. Theory IEEE Trans. 20(2), 284-287 (1974)

26. XTVu, M Di Renzo, P Duhamel, Optimal and low-complexity iterative joint network/channel decoding for the multiple-access relay channel. in the 2011 IEEE International Conference on Acoustics Speech and Signal Processing (ICASSP 2011). Prague, 22-27 May 2011

27. JG Proakis, Digit Communications. McGraw-Hill series in electrical and computer engineering, 4th edition (McGraw-Hill, Boston, 2001). [00025305 John G. Proakis. ill.]

28. A Glavieux, Channel Coding in Communication Networks: From Theory to Turbo Codes (Wiley, New York, 2007)

29. Z Ren, G Wang, Q Chen, H Li, Modelling and simulation of Rayleigh fading, path loss, and shadowing fading for wireless mobile networks. Simulation Modell. Pract. Theory. 19(2), 626-637 (2011)

doi:10.1186/1687-6180-2013-170

Cite this article as: Vu et al:: Multiple-access relaying with network coding: iterative network/channel decoding with imperfect CSI. EURASIP Journal on Advances in Signal Processing 2013 2013:170. 